\title{
Values of the Dedekind Eta Function at Quadratic Irrationalities
}

\author{
Alfred van der Poorten and Kenneth S. Williams
}

Abstract. Let $d$ be the discriminant of an imaginary quadratic field. Let $a, b, c$ be integers such that

$$
b^{2}-4 a c=d, \quad a>0, \quad \operatorname{gcd}(a, b, c)=1 .
$$

The value of $|\eta((b+\sqrt{d}) / 2 a)|$ is determined explicitly, where $\eta(z)$ is Dedekind's eta function

$$
\eta(z)=\mathrm{e}^{\pi \mathrm{i} z / 12} \prod_{m=1}^{\infty}\left(1-\mathrm{e}^{2 \pi \mathrm{imz}}\right) \quad(\mathrm{im}(\mathrm{z})>0) .
$$

\section{Introduction}

The Dedekind eta function $\eta(z)$ is defined for all complex numbers $z=x+i y$ with $y>0$ by

$$
\eta(z):=\mathrm{e}^{\pi \mathrm{iz} / 12} \prod_{m=1}^{\infty}\left(1-\mathrm{e}^{2 \pi \mathrm{imz}}\right)
$$

The basic properties of $\eta(z)$ are given for example in [9, pp. 14-22].

Let $F$ be an imaginary quadratic field. Let $d$ denote the discriminant of $F$. Thus $d$ is a negative integer with $d \equiv 0$ or $1(\bmod 4), F=Q(\sqrt{d})$, and the largest positive integer $m$ such that $\mathrm{m}^{2} \mid \mathrm{d}$ and $\mathrm{d} / \mathrm{m}^{2} \equiv 0$ or $1(\bmod 4)$ is $\mathrm{m}=1$. Let $\mathrm{a}, \mathrm{b}$ and $\mathrm{c}$ be integers satisfying

$$
b^{2}-4 a c=d, \quad a>0, \quad \operatorname{gcd}(a, b, c)=1 .
$$

Such integers exist as we may take

$$
\begin{gathered}
a=1, \quad b=0, \quad c=-d / 4, \quad \text { if } d \equiv 0 \quad(\bmod 4), \\
a=1, \quad b=1, \quad c=(1-d) / 4, \quad \text { if } d \equiv 1 \quad(\bmod 4) .
\end{gathered}
$$

The main result of this paper is the explicit determination of $|\eta((b+\sqrt{d}) / 2 a)|$ (Theorem 9.3). We describe briefly how Theorem 9.3 is proved. Section 2 is devoted to giving

\footnotetext{
Received by the editors July 16, 1998; revised November 19, 1998.

Research of the second author was supported by Natural Sciences and Engineering Research Council of Canada grant A-7233.

AM S subject classification: 11F20, 11E45.

Keywords: Dedekind eta function, quadratic irrationalities, binary quadratic forms, form class group.

(c) Canadian M athematical Society 1999.
} 
the basic definitions, notation and results necessary for the rest of the paper. In Section 3 we prove an explicit formula for the number $P_{K}(n)$ of proper representations of a positive integer $n$ by the class $\mathrm{K}$ of the form class group $\mathrm{H}$ (d) of discriminant $d$ (Proposition 3.1). In Section 4 it is shown (Proposition 4.1) that the following linear combination of the $P_{L}(n)$ $(L \in H(d))$

$$
W_{K}(n):=\frac{1}{W(d)} \sum_{L \in H(d)} f(K, L) P_{L}(n) \quad(\sec (2.23))
$$

is a multiplicative function of $n$ for each $K \in H(d)$. The quantities $w(d)(=2,4$ or 6$)$ and $f(K, L)$ (= a certain root of unity) are defined in (2.9) and (2.21) respectively. In Sections 5 and 6 certain infinite products are examined which are needed in later sections. In Sections 7 and 8 the behaviour of $\sum_{n=1}^{\infty} \frac{W_{k}(n)}{n^{5}}$ as $\rightarrow 1^{+}$is determined (Propositions 7.1, 7.2 and 8.1 ) in terms of the infinite products discussed in Sections 5 and 6. Inverting (1.3) we obtain

$$
P_{K}(n)=\frac{w(d)}{h(d)} \sum_{L \in H(d)} f(L, K)^{-1} W_{L}(n),
$$

see proof of Proposition 9.1. The total number of representations of $n$ by the class $K$ of $H(d)$ is denoted by $R_{K}(n)$. Clearly

$$
R_{K}(n)=\sum_{e^{2} \mid n} P_{K}\left(n / e^{2}\right) \quad(\sec (2.5))
$$

so that by (1.4) and (1.5)

$$
\sum_{n=1}^{\infty} \frac{R_{K}(n)}{n^{s}}=\zeta(2 s) \sum_{n=1}^{\infty} \frac{P_{K}(n)}{n^{s}}=\frac{\zeta(2 s) W(d)}{h(d)} \sum_{L \in H(d)} f(L, K)^{-1} \sum_{n=1}^{\infty} \frac{W_{L}(n)}{n^{s}}
$$

see proof of Proposition 9.1. From (1.6) knowing the behaviour of $\sum_{n=1}^{\infty} \frac{W_{k}(n)}{n^{s}}$ as $s \rightarrow 1^{+}$ for each $K \in H(d)$, we can determine the behaviour of $\sum_{n=1}^{\infty} \frac{R_{K}(n)}{n^{s}}$ as $s \rightarrow 1^{+}$in the form

$$
\sum_{n=1}^{\infty} \frac{R_{K}(n)}{n^{s}}=\frac{2 \pi / \sqrt{|d|}}{s-1}+A(K, d)+o(s-1)
$$

for a certain constant $A(K, d)$ (Proposition 9.1). On the other hand we can obtain a second representation

$$
\sum_{n=1}^{\infty} \frac{R_{k}(n)}{n^{s}}=\frac{2 \pi / \sqrt{|d|}}{s-1}+B(a, b, c)+o(s-1)
$$

for a certain constant $B(a, b, c)$, where $K=[a, b, c]$, by making use of Kronecker's limit formula for $\sum_{\substack{x, y=-\infty \\(x, y) \neq(0,0)}}^{\infty} \frac{1}{\left(a x^{2}+b x y+c y^{2}\right)^{s}}$, see Proposition 9.2. The equality $A(K, d)=B(a, b, c)$ gives Theorem 9.3. 
Two numerical examples are discussed in Section 9. In Section 10 it is shown that the famous Chowla-Selberg formula for $\prod_{[a, b, c] \in H(d)} a^{-1 / 4}|\eta((b+\sqrt{d}) / 2 a)|($ Theorem 10.1) is a simple consequence of Theorem 9.3. In Section 11 it is proved that the Chowla-Selberg formula for genera (Theorem 11.1) due to Williams and Zhang [10] in 1993 is also a consequence of Theorem 9.3. In Section 12 an expression appearing in the evaluation of $|\eta((b+\sqrt{d}) / 2 a)|$ given in Theorem 9.3 is discussed.

\section{Notation}

Let $d$ be the discriminant of an imaginary quadratic field $F$. Let $a, b, c$ be integers satisfying (1.2). Thus

$$
f=f(x, y)=a x^{2}+b x y+c y^{2}
$$

is a positive-definite, primitive, integral, binary quadratic form of discriminant $d$. We call $f$ a form for short and write $f=(a, b, c)$. Let $\Gamma$ denotethe classical modular group, that is,

$$
\Gamma:=\left\{\left(\begin{array}{ll}
r & s \\
t & u
\end{array}\right) \mid r, s, t, u \in Z, \quad r u-s t=1\right\} .
$$

The class of the form $f=(a, b, c)$ is the set of forms

$$
[f]=\left\{f(r x+s y, t x+u y) \mid\left(\begin{array}{ll}
r & s \\
t & u
\end{array}\right) \in \Gamma\right\} .
$$

We write $[a, b, c]$ for $[f]=[(a, b, c)]$. With respect to Gaussian composition the set $H(d)$ of classes of forms of discriminant $d$ is a finite abelian group called the form class group. The number $h(d)$ of classes in $H(d)$ is called the form class number. The identity I of the form class group $\mathrm{H}(\mathrm{d})$ is the class

$$
I=\left\{\begin{array}{lll}
{[1,0,-d / 4],} & \text { if } d \equiv 0 \quad(\bmod 4) \\
{[1,1,(1-d) / 4],} & \text { if } d \equiv 1 \quad(\bmod 4)
\end{array}\right.
$$

and the inverse of the class $[a, b, c] \in H(d)$ is the class $[a, b, c]^{-1}=[a,-b, c] \in H(d)$.

A positive integer $n$ is said to be represented by the form $f=(a, b, c)$ if there exist integers $x$ and $y$ such that

$$
n=a x^{2}+b x y+c y^{2}
$$

A pair ( $x, y)$ of integers satisfying (2.1) is called a representation of $n$ by the form (a, b, c). If $\operatorname{gcd}(x, y)=1$ the representation is said to be proper. As $d<0$ there are only finitely many representations of $n$ by $(a, b, c)$. The number of representations of $n$ by the form $f$ is denoted by $R_{f}(n)$ and the number of proper representations by $P_{f}(n)$. It is well known and easily proved that

$$
R_{f}(n)=\sum_{e^{2} \mid n} P_{f}\left(n / e^{2}\right),
$$


where e runs through the positive integers whose squares divide $n$. If the forms $f_{1}$ and $f_{2}$ belong to the same class $\mathrm{K} \in \mathrm{H}(\mathrm{d})$ then there is a one-to-one correspondence between the representations of $n$ by $f_{1}$ and those of $n$ by $f_{2}$, as well as a one-to-one correspondence between the proper representations of $n$ by $f_{1}$ and those of $n$ by $f_{2}$. Hence if $K \in H(d)$ and $f_{1}, f_{2} \in K$ then $R_{f_{1}}(n)=R_{f_{2}}(n)$ and $P_{f_{1}}(n)=P_{f_{2}}(n)$. Thus we can define the number $R_{K}(n)$ of representations of $n$ by the class of $K$ of $H(d)$ by

$$
R_{K}(n):=R_{f}(n) \quad \text { for any } f \in K
$$

and the number $P_{K}(n)$ of proper representations of $n$ by the class $K$ by

$$
P_{K}(n):=P_{f}(n) \quad \text { for any } f \in K \text {. }
$$

From (2.2), (2.3) and (2.4), we see that

$$
R_{K}(n)=\sum_{e^{2} \mid n} P_{K}\left(n / e^{2}\right)
$$

for any positive integer $n$ and any $K \in H(d)$. We also set

$$
R(n):=\sum_{K \in H(d)} R_{K}(n), \quad P(n):=\sum_{K \in H(d)} P_{K}(n),
$$

so that by (2.5) we have

$$
R(n)=\sum_{e^{2} \mid n} P\left(n / e^{2}\right)
$$

It is known that

$$
R_{K}(1)=P_{K}(1)= \begin{cases}w(d), & \text { if } K=1 \\ 0, & \text { if } K \neq 1\end{cases}
$$

where

$$
w(d):= \begin{cases}6, & \text { if } d=-3 \\ 4, & \text { if } d=-4 \\ 2, & \text { if } d<-4\end{cases}
$$

The definition of the Legendre Jacobi-Kronecker symbol $\left(\frac{d}{k}\right)$ for a discriminant $d$ and a positive integer $k$ is recalled in [5, p. 278]. If $p$ is a prime for which $\left(\frac{d}{p}\right)=-1$ then $R_{K}(p)=P_{K}(p)=0$ for any class $K$ of $H(d)$. If $\left(\frac{d}{p}\right)=+1$ the congruencet $^{2} \equiv d(\bmod 4 p)$ has exactly two solutions satisfying $0 \leq t<2 p$. We let $t$ denote the smaller of these and define the class $K_{p} \in H(d)$ by

$$
K_{p}=\left[p, t,\left(t^{2}-d\right) / 4 p\right]
$$


The prime $\mathrm{p}$ is represented by the classes $\mathrm{K}_{\mathrm{p}}$ and $\mathrm{K}_{\mathrm{p}}^{-1}$ of $\mathrm{H}(\mathrm{d})$ and by no others. If $\left(\frac{\mathrm{d}}{\mathrm{p}}\right)=0$ (equivalently $p \mid d$ ) then $p$ is represented only by the class

$$
K_{p}=K_{p}^{-1}=\left[p, \lambda p,\left(\lambda^{2} p^{2}-d\right) / 4 p\right]
$$

where

$$
\lambda:= \begin{cases}0, & \text { if } p>2, d \equiv 0 \quad(\bmod 4) \\ & \text { or } p=2, d \equiv 8 \quad(\bmod 16) \\ 1, & \text { if } p>2, d \equiv 1 \quad(\bmod 4) \\ & \text { or } p=2, d \equiv 12 \quad(\bmod 16)\end{cases}
$$

Moreover

$$
R_{K}(p)=P_{K}(p)= \begin{cases}0, & \text { if }\left(\frac{d}{p}\right)=-1 \\ & \text { or }\left(\frac{d}{p}\right)=0 \text { or } 1 \text { and } K \neq K_{p}, K_{p}^{-1}, \\ w(d), & \text { if }\left(\frac{d}{p}\right)=1 \text { and } K=K_{p} \neq K_{p}^{-1} \\ & \text { or }\left(\frac{d}{p}\right)=1 \text { and } K=K_{p}^{-1} \neq K_{p} \\ & \text { or }\left(\frac{d}{p}\right)=0 \text { and } K=K_{p}\left(=K_{p}^{-1}\right) \\ 2 w(d), & \text { if }\left(\frac{d}{p}\right)=1 \text { and } K=K_{p}=K_{p}^{-1} .\end{cases}
$$

M ore generally in Section 3 we give an explicit formula for $P_{K}(n)$ for any positive integer $n$ and any class $K$ of $H(d)$, see Proposition 3.1. This formula for $P_{K}(n)$ is given in terms of the quantity $N_{K}(n)$ defined in Definition 2.1.

Definition 2.1 Let $n$ be a positive integer. Let $K \in H(d)$. Suppose first that $n>1$. Let $n=p_{1}^{a_{1}} \ldots p_{r}^{a_{r}}$ be the prime power decomposition of $n$, that is, $p_{1}, \ldots, p_{r}$ are $r(\geq 1)$ distinct primes and $a_{1}, \ldots, a_{r}$ are positive integers. If $\left(\frac{d}{p_{i}}\right)=-1$ for some $(1 \leq i \leq r)$ we set $N_{K}(n)=0$. If $\left(\frac{d}{p_{i}}\right)=0$ or 1 for every $i(1 \leq i \leq r)$ we set

$$
\begin{aligned}
\mathrm{N}_{\mathrm{K}}(\mathrm{n}):= & \text { number of }\left(\varepsilon_{1}, \ldots, \varepsilon_{\mathrm{r}}\right) \in\{-1,1\}^{\mathrm{r}} \text { such that } \\
& \left(\mathrm{K}_{\mathrm{p}_{1}}\right)^{\mathrm{a}_{1} \varepsilon_{1}} \ldots\left(\mathrm{K}_{\mathrm{p}_{\mathrm{r}}}\right)^{\mathrm{a}_{\mathrm{r}} \varepsilon_{\mathrm{r}}}=\mathrm{K} .
\end{aligned}
$$

Now suppose that $n=1$. In this case we set

$$
\mathrm{N}_{\mathrm{K}}(1):= \begin{cases}1, & \text { if } \mathrm{K}=\mathrm{I} \\ 0, & \text { if } \mathrm{K} \neq \mathrm{I}\end{cases}
$$

We note that

$$
\sum_{K \in H(d)} N_{K}(n)=2^{r}
$$


We let the type of the finite abelian group $\mathrm{H}(\mathrm{d})$ be $\left(\mathrm{h}_{1}, \ldots, \mathrm{h}_{\ell}\right)$. The positive integers $h_{1}, \ldots, h_{\ell}$ are called the invariants of $H(d)$ and are such that

$$
h_{1} \cdots h_{\ell}=h(d), \quad 1<h_{1}\left|h_{2}\right| \cdots \mid h_{\ell} .
$$

Moreover there exist $A_{1}, \ldots, A_{\ell} \in H(d)$ such that $\operatorname{ord}\left(A_{i}\right)=h_{i}(i=1, \ldots, \ell)$ and for each $\mathrm{K} \in \mathrm{H}(\mathrm{d})$ there exist unique integers $\mathrm{k}_{1}, \ldots, \mathrm{k}_{\ell}$ such that

$$
K=A_{1}^{k_{1}} \cdots A_{\ell}^{k_{\ell}}, \quad 0 \leq k_{j}<h_{j} \quad(j=1, \ldots, \ell) .
$$

We fix the choice of the generators $A_{1}, \ldots, A_{\ell}$ of $H(d)$ once and for all. The nonnegative integer $k_{j}$ is called the index of $K$ with respect to the $j$-th element of the ordered set of generators $\mathcal{A}=\left\{\mathrm{A}_{1}, \ldots, \mathrm{A}_{\ell}\right\}$ and is written

$$
\operatorname{ind}_{A_{j}}(K)=k_{j} \quad(j=1, \ldots, \ell) .
$$

For $K, L, M \in H(d)$ we set

$$
[K, L]:=\sum_{j=1}^{\ell} \frac{\operatorname{ind}_{A_{j}}(K) \operatorname{ind}_{A_{j}}(L)}{h_{j}} .
$$

We note that $[K, L]=[L, K],[K, I]=0,[K L, M] \equiv[K, M]+[L, M](\bmod 1)$, and $\left[K^{r}, L^{s}\right] \equiv r s[K, L](\bmod 1)$ for integers $r$ and $s$. Then we define

$$
f(K, L):=e^{2 \pi i[K, L]} .
$$

If it is important to indicate the basis $\mathcal{A}$ we write $[K, L]_{\mathcal{A}}$ for $[K, L]$ and $f_{\mathcal{A}}(K, L)$ for $f(K, L)$. Clearly $f(K, L)=f(L, K), f(K, I)=1, f(K L, M)=f(K, M) f(L, M)$ and $f\left(K^{r}, L^{s}\right)=f(K, L)^{r s}$ for any integers $r$ and $s$.

Simple calculations show that

$$
\left\{\begin{array}{l}
\sum_{M \in H(d)} f(K, M)= \begin{cases}h(d), & \text { if } K=l, \\
0, & \text { if } K \neq l,\end{cases} \\
\sum_{M \in H(d)} f(K, M) f(M, L)^{-1}= \begin{cases}h(d), & \text { if } K=L, \\
0, & \text { if } K \neq L .\end{cases}
\end{array}\right.
$$

Next, for a positive integer $n$ and $K \in H(d)$, we define

$$
W_{K}(n):=\frac{1}{W(d)} \sum_{L \in H(d)} f(K, L) P_{L}(n)
$$

We note that

$$
W_{K}(1)=\frac{1}{W(d)} \sum_{L \in H(d)} f(K, L) P_{L}(1)=f(K, I)=1
$$


and that

$$
W_{\mid}(n)=\frac{1}{W(d)} \sum_{L \in H(d)} f(I, L) P_{L}(n)=\frac{1}{W(d)} \sum_{L \in H(d)} P_{L}(n)=\frac{P(n)}{W(d)}
$$

We will also use the following notation:

$$
\begin{aligned}
\tau(\mathrm{n}):= & \text { number of distinct prime divisors of the positive } \\
& \text { integer } \mathrm{n} \text { (so that } \tau(1)=0 \text { ), }
\end{aligned}
$$

$$
\mathrm{n}^{*}:=\text { radical of } \mathrm{n}=\prod_{\mathrm{p} \mid \mathrm{n}} \mathrm{p} \quad\left(\text { so that } 1^{*}=1\right)
$$

$$
\begin{aligned}
\gamma:=\text { Euler's constant } & =\lim _{n \rightarrow \infty}\left(1+\frac{1}{2}+\cdots+\frac{1}{n}-\log n\right) \\
& =0.57721566 \text { (approx) }
\end{aligned}
$$

$$
\begin{aligned}
\zeta(\mathrm{s}):=\text { Riemann zeta function } & =\sum_{\mathrm{n}=1}^{\infty} \frac{1}{\mathrm{n}^{s}} \\
& =\prod_{\mathrm{p}}\left(1-\frac{1}{\mathrm{p}^{s}}\right)^{-1}(\mathrm{~s}>1) \quad\left(\text { so that } \zeta(2)=\pi^{2} / 6\right),
\end{aligned}
$$

$$
L(s, d):=\text { Dirichlet's L-series for discriminant d }
$$

$$
\begin{aligned}
&= \sum_{n=1}^{\infty} \frac{\left(\frac{d}{n}\right)}{n^{s}}(s>0), \\
& \Gamma(x):= \text { gamma function }=\int_{0}^{\infty} t^{x-1} e^{-t} d t \quad(x>0) \\
&(\text { so that } \Gamma(n)=(n-1) !), \\
& t_{j}(d):= \prod_{\substack{p \\
\left(\frac{d}{p}\right)=j}}\left(1-\frac{1}{p^{2}}\right) \quad(j=-1,0,1) \\
&\left(\text { so that } t_{-1}(d) t_{0}(d) t_{1}(d)=\prod_{p}\left(1-\frac{1}{p^{2}}\right)=\frac{6}{\pi^{2}}\right),
\end{aligned}
$$




$$
\ell(K, d):=\prod_{\substack{p \\\left(\frac{d}{p}\right)=0}}\left(1+\frac{f\left(K, K_{p}\right)}{p}\right) \quad(K \in H(d)) .
$$

With regard to (2.33), we note that writing $(m, n)$ for $\operatorname{gcd}(m, n)$ from now on

$$
\begin{aligned}
\left(\frac{d}{p}\right)=0 & \Rightarrow K_{p}=K_{p}^{-1} \\
& \Rightarrow K_{p}=\prod_{j=1}^{\ell} A_{j}^{\left(2, h_{j}\right)}, \quad c_{j}=0, \ldots,\left(2, h_{j}\right)-1, \quad j=1, \ldots, \ell, \\
& \Rightarrow f\left(K, K_{p}\right)=e^{2 \pi i \sum_{j=1}^{\ell} \frac{h_{j} c_{j}}{\left(2, h_{j}\right.} K_{j} / h_{j}}=(-1)^{\sum_{j=1}^{\ell} \frac{2}{\left(2, h_{j}\right.} K_{j} c_{j}}= \pm 1,
\end{aligned}
$$

so that $\ell(K, d)$ is real. Also

$$
\begin{aligned}
\ell\left(K^{-1}, d\right) & =\prod_{\substack{p \\
\left(\frac{d}{p}\right)=0}}\left(1+\frac{f\left(K^{-1}, K_{p}\right)}{p}\right) \\
& =\prod_{\substack{p \\
\left(\frac{d}{p}\right)=0}}\left(1+\frac{f\left(K, K_{p}\right)^{-1}}{p}\right) \\
& =\prod_{\substack{p \\
\left(\frac{d}{p}\right)=0}}\left(1+\frac{f\left(K, K_{p}\right)}{p}\right)
\end{aligned}
$$

as $\mathrm{f}\left(\mathrm{K}, \mathrm{K}_{\mathrm{p}}\right)= \pm 1$, so that

$$
\ell\left(K^{-1}, d\right)=\ell(K, d)
$$

\section{Formula for $P_{k}(n)$}

We prove

Proposition 3.1 Let $n$ bea positive integer and let $K \in H(d)$. Then

$$
P_{K}(n)=\frac{w(d)}{2^{\tau(n)}}\left(\frac{d}{n / n^{*}}\right)\left(\sum_{g \mid n}^{\prime}\left(\frac{d}{g}\right)\right) N_{K}(n),
$$

where the prime (') indicates that the (positive) divisors $g$ of $n$ are restricted to be squarefree.

Proof When $n=1$ the right hand side of the asserted formula is $w(d) N_{K}(1)$, which is equal to $P_{K}(1)$ by (2.8) and (2.15). Thus we may assume that $n>1$. 
If $n$ has a prime factor $q$ such that $q^{2}|n, q| d$ then it is easy to show that $n$ cannot be represented properly by any form of discriminant $d$ so that $P_{k}(n)=0$. Set $n=q^{\beta} n_{1}$, where $\mathrm{q} \nmid \mathrm{n}_{1}$ and $\beta \geq 2$. Then

$$
\left(\frac{d}{n / n^{*}}\right)=\left(\frac{d}{q^{\beta-1} n_{1} / n_{1}^{*}}\right)=\left(\frac{d}{q}\right)^{\beta-1}\left(\frac{d}{n_{1} / n_{1}^{*}}\right)=0,
$$

showing that the asserted formula holds in this case.

Further, if $n$ has a prime factor $r$ such that $\left(\frac{d}{r}\right)=-1$ then again it is easy to show that $n$ is not properly represented by any form of discriminant $d$ so that $P_{K}(n)=0$. Set $n=r^{\gamma} n_{1}$, where $\nmid \mathrm{n}_{1}$ and $\gamma \geq 1$. Then

$$
\sum_{g \mid n}^{\prime}\left(\frac{d}{g}\right)=\left(1+\left(\frac{d}{r}\right)\right) \sum_{g_{1} \mid n_{1}}^{\prime}\left(\frac{d}{g_{1}}\right)=0
$$

so the asserted formula works in this case too.

Hence we may suppose that $n$ has a prime factorization of the form

$$
\mathrm{n}=\mathrm{p}_{1}^{\mathrm{a}_{1}} \cdots \mathrm{p}_{\mathrm{r}}^{\mathrm{a}_{\mathrm{r}}} \mathrm{q}_{1} \cdots \mathrm{q}_{\mathrm{s}}
$$

where the $p_{i}(i=1, \ldots, r)$ are distinct primes with $\left(\frac{d}{p_{i}}\right)=1$, the $q_{i}(i=1, \ldots, s)$ are distinct primes with $\left(\frac{d}{q_{i}}\right)=0$ (equivalently $q_{i} \mid d$ ), and the $a_{i}$ are positive integers. In the ring of integers $O_{F}$ of $F=Q(\sqrt{d})$ we have (see for example [1, p. 142])

$$
\mathrm{p}_{\mathrm{i}} \mathrm{O}_{\mathrm{F}}=\mathrm{P}_{\mathrm{i}} \mathrm{P}_{\mathrm{i}}^{\prime} \quad(\mathrm{i}=1, \ldots, \mathrm{r})
$$

where $\mathrm{P}_{\mathrm{i}}$ and $\mathrm{P}_{\mathrm{i}}^{\prime}$ are distinct conjugate prime ideals with $\mathrm{N}\left(\mathrm{P}_{\mathrm{i}}\right)=\mathrm{N}\left(\mathrm{P}_{\mathrm{i}}^{\prime}\right)=\mathrm{p}_{\mathrm{i}}$, and

$$
\mathrm{q}_{\mathrm{i}} \mathrm{O}_{\mathrm{F}}=\mathrm{Q}_{\mathrm{i}}^{2} \quad(\mathrm{i}=1, \ldots, \mathrm{s}),
$$

where $Q_{i}$ is a self-conjugate prime ideal with $N\left(Q_{i}\right)=q_{i}$. Thus the prime ideal decomposition of the principal ideal $\mathrm{nO}_{\mathrm{F}}$ is

$$
n \mathrm{O}_{\mathrm{F}}=\mathrm{P}_{1}^{\mathrm{a}_{1}} \mathrm{P}_{1}^{\prime \mathrm{a}_{1}} \ldots \mathrm{P}_{\mathrm{r}}^{\mathrm{a}_{\mathrm{r}}} \mathrm{P}_{\mathrm{r}}^{\prime \mathrm{a}_{\mathrm{r}}} Q_{1}^{2} \ldots \mathrm{Q}_{\mathrm{s}}^{2} .
$$

A nonzero ideal $\mathrm{A}$ of $\mathrm{O}_{\mathrm{F}}$ is said to be integerfree if $\mathrm{KO}_{\mathrm{F}} \mid \mathrm{A}$, where $\mathrm{k}$ is an integer, implies that $\mathrm{k}= \pm 1$. From the prime ideal decomposition of $\mathrm{nO}_{\mathrm{F}}$, we see that all integerfree ideals $\mathrm{A}$ of $\mathrm{O}_{\mathrm{F}}$ with norm $\mathrm{n}$ are given by

$$
A=P_{1}^{a_{1}} P_{1}^{\prime a_{1}-u_{1}} \ldots P_{r}^{u_{r}} P_{r}^{\prime a_{r}-u_{r}} Q_{1} \ldots Q_{s},
$$

where each $u_{i}=0$ or $a_{i}$. For $K=[a, b, c] \in H(d)$ we set

$$
I_{K}(n):=\text { number of integerfree ideals } A \text { of } O_{F} \text { with }
$$

$$
N(A)=n \text { and } \bar{A}=\left[\overline{a, \frac{-b+\sqrt{d}}{2}}\right] \text {. }
$$


Here $\bar{A}$ denotes the class of the ideal $A$ in the ideal class group. We note that $I_{K}(n)$ is welldefined for if $K=[a, b, c]=\left[a^{\prime}, b^{\prime}, c^{\prime}\right]$ then $\left[\overline{a, \frac{-b+\sqrt{d}}{2}}\right]=\left[\overline{a^{\prime}, \frac{-b^{\prime}+\sqrt{d}}{2}}\right]$, see for example [2, Theorem 7.7]. From (3.1) and (3.2) we deduce that

$$
\begin{aligned}
& I_{K}(n)=\text { number of } r \text {-tuples }\left(u_{1}, \ldots, u_{r}\right) \text { with } \\
& \text { each } u_{i}=0 \text { or } a_{i} \text { such that } \\
& \quad \bar{P}_{1}^{u_{1}} \bar{P}_{1}^{\prime a_{1}-u_{i}} \ldots \bar{P}_{r}^{u_{r}} \bar{P}_{r}^{\prime a_{r}-u_{r}} \bar{Q}_{1} \ldots \bar{Q}_{s}=\left[\overline{a, \frac{-b+\sqrt{d}}{2}}\right] .
\end{aligned}
$$

As $P_{i} P_{i}^{\prime}$ and $P_{i}^{h(d)}$ are both principal ideals in $O_{F}$, we have

$$
\overline{P_{i}} \overline{P_{i}^{\prime}}=\overline{P_{i} P_{i}^{\prime}}=\overline{P_{i}^{h(d)}}=\left(P_{i}\right)^{h(d)},
$$

so that

$$
\overline{P_{i}^{\prime}}=\left(\bar{P}_{i}\right)^{h(d)-1}, \quad i=1, \ldots, r .
$$

Set

$$
\varepsilon_{\mathrm{i}}= \begin{cases}1, & \text { if } u_{i}=a_{i} \\ -1, & \text { if } u_{i}=0\end{cases}
$$

Hence

$$
\begin{aligned}
P_{i}^{u_{i}} P_{i}^{\prime a_{i}-u_{i}} & =\bar{P}_{i}^{u_{i}+(h(d)-1)\left(a_{i}-u_{i}\right)} \\
& = \begin{cases}P_{i}^{a_{i}}, & \text { if } u_{i}=a_{i}, \\
\left(\bar{P}_{i}\right)^{-a_{i}}, & \text { if } u_{i}=0,\end{cases} \\
& =\bar{P}_{i}^{\varepsilon_{i} a_{i}} .
\end{aligned}
$$

Thus

$$
\begin{array}{r}
\mathrm{I}_{\mathrm{K}}(\mathrm{n})=\text { number of }\left(\varepsilon_{1}, \ldots, \varepsilon_{\mathrm{r}}\right) \in\{-1,1\}^{\mathrm{r}} \text { such that } \\
\qquad \bar{P}_{1}^{\varepsilon_{1} \mathrm{a}_{1}} \ldots \overline{\mathrm{P}}_{\mathrm{r}}^{\varepsilon_{\mathrm{r}} \mathrm{a}_{\mathrm{r}}} \overline{\mathrm{Q}}_{1} \ldots \overline{\mathrm{Q}}_{\mathrm{s}}=\left[\mathrm{a}, \frac{-\mathrm{b}+\sqrt{\mathrm{d}}}{2}\right] .
\end{array}
$$

As $Q_{i}^{2}$ is principal, $\bar{Q}_{i}=\bar{Q}_{i}^{-1}$ so

$$
\begin{aligned}
I_{K}(n)= & \frac{1}{2^{s}} \text { number of }\left(\varepsilon_{1}, \ldots, \varepsilon_{r+s}\right) \in\{-1,1\}^{r+s} \text { such that } \\
& P_{1}^{\varepsilon_{1} a_{1}} \ldots P_{r}^{\varepsilon_{r} a_{r}} \bar{Q}_{1}^{\varepsilon_{r+1}} \ldots \bar{Q}_{s}^{\varepsilon_{r+s}}=\left[\overline{a, \frac{-b+\sqrt{d}}{2}}\right] .
\end{aligned}
$$


Let $\alpha$ be the isomorphism between the form class group and the ideal class group given by

$$
\alpha([a, b, c])=\left[\overline{a, \frac{-b+\sqrt{d}}{2}}\right],
$$

see for example [2, Theorem 7.7]. Then

$$
\alpha\left(\mathrm{K}_{\mathrm{p}_{\mathrm{i}}}\right)=\alpha\left(\left[\mathrm{p}_{\mathrm{i}}, \mathrm{t}_{\mathrm{i}},\left(\mathrm{t}_{\mathrm{i}}^{2}-\mathrm{d}\right) / 4 \mathrm{p}_{\mathrm{i}}\right]\right)=\left[\overline{\mathrm{p}_{\mathrm{i}}, \frac{-\mathrm{t}_{\mathrm{i}}+\sqrt{\mathrm{d}}}{2}}\right]=\overline{\mathrm{P}}_{\mathrm{i}}
$$

and

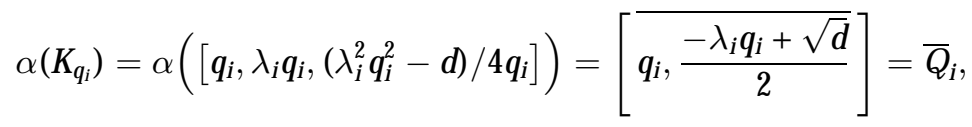

see for example [1, pp. 144-145], so that

$$
\begin{aligned}
\mathrm{I}_{\mathrm{K}}(\mathrm{n})= & \frac{1}{2^{\mathrm{s}}} \text { number of }\left(\varepsilon_{1}, \ldots, \varepsilon_{\mathrm{r}+\mathrm{s}}\right) \in\{-1,1\}^{\mathrm{r}+\mathrm{s}} \text { such that } \\
& \mathrm{K}_{\mathrm{p}_{1}}^{\varepsilon_{1} \mathrm{a}_{1}} \ldots \mathrm{K}_{\mathrm{p}_{\mathrm{r}}}^{\varepsilon_{\mathrm{r}} \mathrm{r}_{\mathrm{r}}} \mathrm{K}_{\mathrm{q}_{1}}^{\varepsilon_{\mathrm{r}+1}} \ldots \mathrm{K}_{\mathrm{q}_{\mathrm{s}}}^{\varepsilon_{\mathrm{r}+\mathrm{s}}}=\mathrm{K} \\
= & \frac{1}{2^{\mathrm{s}}} \mathrm{N}_{\mathrm{K}}(\mathrm{n}) .
\end{aligned}
$$

Now each integerfree ideal $A$ of $O_{F}$ with $N(A)=n$ and $\bar{A}=\left[\overline{a, \frac{-b+\sqrt{d}}{2}}\right]$ gives rise to exactly $w(d)$ proper representations of $n$ by $K=[a, b, c]$, see for example [2, pp. 137-142], so that

$$
P_{K}(n)=w(d) I_{K}(n)
$$

Thus

$$
\begin{aligned}
P_{K}(n)= & \frac{w(d)}{2^{s}} N_{K}(n) \\
= & \frac{w(d)}{2^{r+s}} 2^{r} N_{K}(n) \\
= & \frac{w(d)}{2^{r+s}}\left(\frac{d}{p_{1}^{a_{1}-1} \cdots p_{r}^{a_{r}}-1}\right) \prod_{i=1}^{r}\left(1+\left(\frac{d}{p_{i}}\right)\right) \prod_{j=1}^{s}\left(1+\left(\frac{d}{q_{i}}\right)\right) N_{K}(n) \\
& \left(a s\left(\frac{d}{p_{i}}\right)=1,\left(\frac{d}{q_{j}}\right)=0\right) \\
= & \frac{w(d)}{2^{\tau(n)}}\left(\frac{d}{n / n^{*}}\right) \sum_{g \mid n}^{\prime}\left(\frac{d}{g}\right) N_{K}(n)
\end{aligned}
$$

as asserted. This completes the proof of Proposition 3.1. 


\section{$4 W_{K}(n)$ is a Multiplicative Function of $n$}

In this section we use Proposition 3.1 to prove

Proposition 4.1 Let $n$ bea positiveinteger and let $K \in H(d)$. Then $W_{K}(n)$ is a multiplicative function of $n$.

Proof Let $n_{1}$ and $n_{2}$ be positive integers with $\left(n_{1}, n_{2}\right)=1$. It follows immediately from Definition 2.1 that for all $K \in H(d)$

$$
N_{K}\left(n_{1} n_{2}\right)=\sum_{K_{1} K_{2}=K} N_{K_{1}}\left(n_{1}\right) N_{K_{2}}\left(n_{2}\right),
$$

where the sum is over all pairs $\left(\mathrm{K}_{1}, \mathrm{~K}_{2}\right)$ of classes of $\mathrm{H}(\mathrm{d})$ with $\mathrm{K}_{1} \mathrm{~K}_{2}=\mathrm{K}$.

From (2.23) and Proposition 3.1, we obtain

$$
W_{K}(n)=\frac{1}{2^{\tau(n)}}\left(\frac{d}{n / n^{*}}\right)\left(\sum_{g \mid n}^{\prime}\left(\frac{d}{g}\right)\right) \sum_{L \in H(d)} f(K, L) N_{L}(n) .
$$

Since each of $\frac{1}{2^{\tau(n)}},\left(\frac{d}{n / n^{*}}\right), \sum_{g \mid n}^{\prime}\left(\frac{d}{g}\right)$ is a multiplicative function of $n$, to prove that $W_{K}(n)$ is a multiplicative function of $n$, it suffices to show that $\sum_{L \in H(d)} f(K, L) N_{L}(n)$ is a multiplicative function of $\mathrm{n}$. We have

$$
\begin{aligned}
& \sum_{L \in H(d)} f(K, L) N_{L}\left(n_{1} n_{2}\right) \\
& =\sum_{L \in H(d)} f(K, L) \sum_{L_{1} L_{2}=L} N_{L_{1}}\left(n_{1}\right) N_{L_{2}}\left(n_{2}\right) \\
& =\sum_{L \in H(d)} \sum_{L_{1} L_{2}=L} f\left(K, L_{1} L_{2}\right) N_{L_{1}}\left(n_{1}\right) N_{L_{2}}\left(n_{2}\right) \\
& =\sum_{L_{1} \in H(d)} \sum_{L_{2} \in H(d)} f\left(K, L_{1}\right) f\left(K, L_{2}\right) N_{L_{1}}\left(n_{1}\right) N_{L_{2}}\left(n_{2}\right) \\
& =\sum_{L_{1} \in H(d)} f\left(K, L_{1}\right) N_{L_{1}}\left(n_{1}\right) \sum_{L_{2} \in H(d)} f\left(K, L_{2}\right) N_{L_{2}}\left(n_{2}\right),
\end{aligned}
$$

which completes the proof of

$$
W_{K}\left(n_{1} n_{2}\right)=W_{K}\left(n_{1}\right) W_{K}\left(n_{2}\right), \quad\left(n_{1}, n_{2}\right)=1
$$




\section{Estimation of a Certain Infinite Product}

Our aim in this section is to prove the following result. We make use of the ideas in [4, pp. 346-353].

Proposition 5.1 Let $K \in H(d)$. Let $\omega$ be a complex number with $|\omega|=1$. Then there exists a nonzero complex number $\mathrm{C}(\mathrm{K}, \mathrm{d}, \omega)$ depending only on $\mathrm{K}, \mathrm{d}$ and $\omega$ such that

$$
\prod_{\substack{p \\\left(\frac{d}{p}\right)=1 \\ K_{p}=K}}\left(1-\frac{\omega}{p^{s}}\right)=(s-1)^{\omega / 2 h(d)} C(K, d, \omega)(1+o(s-1)), \quad \text { as } s \rightarrow 1^{+},
$$

where $\mathrm{p}$ runs through prime numbers.

This proposition will be used in the proof of Proposition 6.1. In order to prove Proposition 5.1 we require a number of lemmas. For $x \in R$ and $K \in H(d)$ we set

$$
\begin{gathered}
\pi_{\mathrm{K}, \mathrm{d}}(\mathrm{x}):=\sum_{\substack{\mathrm{p} \leq \mathrm{x} \\
\mathrm{K}_{\mathrm{p}}=\mathrm{K}}} 1, \\
\theta_{\mathrm{K}, \mathrm{d}}(\mathrm{x}):=\sum_{\substack{\mathrm{p} \leq \mathrm{x} \\
\mathrm{K}_{\mathrm{p}}=\mathrm{K}}} \log \mathrm{p}, \\
\kappa_{\mathrm{K}, \mathrm{d}}(\mathrm{x}):=\sum_{\substack{p \leq x \\
K_{\mathrm{p}}=\mathrm{K}}} \frac{\log \mathrm{p}}{\mathrm{p}}, \\
\lambda_{\mathrm{K}, \mathrm{d}}(\mathrm{x}):=\sum_{\substack{p \leq x \\
K_{p}=\mathrm{K}}} \frac{1}{\mathrm{p}},
\end{gathered}
$$

where $p$ runs through prime numbers.

Lemma 5.2 Let $K \in H(d)$. Then

$$
\pi_{K, d}(x)=\frac{1}{2 h(d)} \frac{x}{\log x}+O_{k, d}\left(\frac{x}{\log ^{2} x}\right),
$$

where the constant implied by the 0 -symbol depends on $\mathrm{K}$ and $\mathrm{d}$, and not on $\mathrm{x}$.

Proof From the prime ideal theorem with remainder for ideal classes, see for example [7, Corollary (i), p. 369], and the relationship between the ideal classes in $F=Q(\sqrt{d})$ and the form classes of $\mathrm{H}(\mathrm{d})$, we have

$$
\pi_{K, d}(x)=\frac{1}{2 h(d)} \ell i(x)+O_{K, d}\left(x e^{-b(K, d) \sqrt{\log x}}\right),
$$


for some positive number $b(K, d)$ depending only on $K$ and $d$. As $\ell i(x)=\frac{x}{\log x}+O\left(\frac{x}{\log ^{2} x}\right)$ and $e^{-b(K, d) \sqrt{\log x}}=O_{K, d}\left(\frac{1}{\log ^{2} x}\right)$, the asserted result follows.

Lemma 5.3 Let $\mathrm{K} \in \mathrm{H}(\mathrm{d})$. Then

$$
\theta_{K, d}(x)=\frac{1}{2 h(d)} x+O_{K, d}\left(\frac{x}{\log x}\right) .
$$

Proof By partial summation we have

$$
\theta_{\mathrm{K}, \mathrm{d}}(\mathrm{x})=\pi_{\mathrm{K}, \mathrm{d}}(\mathrm{x}) \log \mathrm{x}-\int_{2}^{\mathrm{x}} \frac{\pi_{\mathrm{K}, \mathrm{d}}(\mathrm{t})}{\mathrm{t}} \mathrm{dt}, \quad \mathrm{x} \geq 2,
$$

see for example [4, Theorem 421, p. 346]. The result now follows on using Lemma 5.2.

Lemma 5.4 Let $K \in K(d)$. Then

$$
\kappa_{K, d}(x)=\frac{1}{2 h(d)} \log x+O_{K, d}(\log \log x) .
$$

Proof By partial summation we have

$$
\kappa_{\mathrm{K}, \mathrm{d}}(\mathrm{x})=\frac{\theta_{\mathrm{K}, \mathrm{d}}(\mathrm{x})}{\mathrm{x}}+\int_{2}^{\mathrm{x}} \frac{\theta_{\mathrm{K}, \mathrm{d}}(\mathrm{t})}{\mathrm{t}^{2}} \mathrm{dt} .
$$

The result follows on using Lemma 5.3.

Lemma 5.5 Let $K \in H(d)$. Then there exists a constant $c(K, d)$ depending only on $K$ and $d$ such that

$$
\lambda_{K, d}(x)=\frac{1}{2 h(d)} \log \log x+c(K, d)+O_{K, d}\left(\frac{1}{\log \log x}\right) .
$$

Proof Set

$$
\kappa_{\mathrm{K}, \mathrm{d}}(\mathrm{x})=\frac{1}{2 \mathrm{~h}(\mathrm{~d})} \log \mathrm{x}+\tau_{\mathrm{K}, \mathrm{d}}(\mathrm{x}) .
$$

By Lemma 5.4 we have $\tau_{k, d}(x)=0_{K, d}(\log \log x)$. Next, by partial summation, we have

$$
\lambda_{\mathrm{K}, \mathrm{d}}(\mathrm{x})=\frac{\kappa_{\mathrm{K}, \mathrm{d}}(\mathrm{x})}{\log \mathrm{x}}+\int_{2}^{\mathrm{x}} \frac{\kappa_{\mathrm{K}, \mathrm{d}}(\mathrm{t})}{\mathrm{t} \log ^{2} \mathrm{t}} \mathrm{dt} .
$$

Appealing to Lemma 5.4, we obtain

$$
\lambda_{k, d}(x)=\frac{1}{2 h(d)}+O_{K, d}\left(\frac{\log \log x}{\log x}\right)+\frac{1}{2 h(d)}(\log \log x-\log \log 2)+\int_{2}^{x} \frac{\tau_{k, d}(t)}{t \log ^{2} t} d t .
$$

As $\tau_{K, d}(t)=O_{K, d}(\log \log t)$ the integrals $\int_{2}^{\infty} \frac{\tau_{K, d}(d)}{t \log ^{2} t} d t$ and $\int_{x}^{\infty} \frac{\tau_{k, d}(t)}{t \log ^{2} t} d t$ are convergent. Moreover

$$
\int_{x}^{\infty} \frac{\tau_{k, d}(t) d t}{t \log ^{2} t}=O_{k, d}\left(\frac{1}{\log \log x}\right)
$$


so

$$
\lambda_{K, d}(x)=\frac{1}{2 h(d)} \log \log x+c(K, d)+O_{K, d}\left(\frac{1}{\log \log x}\right),
$$

with

$$
c(K, d)=\frac{1}{2 h(d)}(1-\log \log 2)+\int_{2}^{\infty} \frac{\left(\kappa_{K, d}(t)-\frac{1}{2 h(d)} \log t\right)}{t \log ^{2} t} d t .
$$

Lemma 5.6 Let $K \in H(d)$. Then

$$
\sum_{\substack{p \\ K_{p}=K}} \frac{1}{p^{s}}=-\frac{1}{2 h(d)} \log (s-1)+\left(c(K, d)-\frac{\gamma}{2 h(d)}\right)+o(s-1),
$$

ass $\rightarrow 1^{+}$.

Proof Let $\delta$ be a real number satisfying $0<\delta<1 / 4$. By partial summation we have

$$
\sum_{\substack{p \leq x \\ k_{p}=k}} \frac{1}{p^{1+\delta}}=\frac{\lambda_{k, d}(x)}{x^{\delta}}+\delta \int_{2}^{x} \frac{\lambda_{k, d}(t)}{t^{1+\delta}} d t, \quad x \geq 2
$$

Let $x \rightarrow+\infty$. By Lemma 5.5 we obtain

$$
\sum_{\substack{p \\ K_{p}=K}} \frac{1}{p^{1+\delta}}=\delta \int_{2}^{\infty} \frac{\lambda_{K, d}(t)}{t^{1+\delta}} d t
$$

Set

$$
\lambda_{K, d}(x)=\frac{1}{2 h(d)} \log \log x+c(K, d)+E_{K, d}(x) .
$$

By Lemma 5.5 we have $E_{k, d}(x)=0_{k, d}\left(\frac{1}{\log \log x}\right)$, say

$$
\left|E_{K, d}(x)\right| \leq \frac{e(K, d)}{\log \log x}, \quad x>e,
$$

for some positive number $e(K, d)$. Then

$$
\sum_{\substack{p \\ K_{p}=K}} \frac{1}{p^{1+\delta}}=\frac{\delta}{2 h(d)} \int_{2}^{\infty} \frac{\log \log t}{t^{1+\delta}} d t+\frac{c(K, d)}{2^{\delta}}+\delta \int_{2}^{\infty} \frac{E_{K, d}(t)}{t^{1+\delta}} d t
$$

as $\delta \int_{2}^{\infty} \frac{d \mathrm{t}}{\mathrm{t}^{1+\delta}}=\frac{1}{2^{\delta}}$. Now

$$
\left|\int_{1}^{2} \frac{\log \log t}{t^{1+\delta}} d t\right| \leq \int_{1}^{2} \frac{|\log \log t|}{t} d t=\text { constant }
$$


so that

$$
\delta \int_{1}^{2} \frac{\log \log t}{\mathrm{t}^{1+\delta}} \mathrm{dt}=0(\delta) .
$$

Further, putting $\mathrm{t}=\mathrm{e}^{\mathrm{u} / \delta}$, we obtain

$$
\begin{aligned}
\delta \int_{1}^{\infty} \frac{\log \log t}{\mathrm{t}^{1+\delta}} d t & =\int_{0}^{\infty} \mathrm{e}^{-\mathrm{u}} \log \left(\frac{\mathrm{u}}{\delta}\right) \mathrm{du} \\
& =\int_{0}^{\infty} \mathrm{e}^{-\mathrm{u}} \log \mathrm{u} \mathrm{du}-\log \delta \int_{0}^{\infty} \mathrm{e}^{-\mathrm{u}} \mathrm{du} \\
& =-\gamma-\log \delta,
\end{aligned}
$$

as

$$
\int_{0}^{\infty} e^{-u} \log u d u=-\gamma
$$

see for example [3, p. 602]. Hence

$$
\delta \int_{2}^{\infty} \frac{\log \log t}{t^{1+\delta}} d t=-\gamma-\log \delta+0(\delta) .
$$

Now set $\mathrm{T}=\mathrm{e}^{\mathrm{I} / \sqrt{\delta}}$ so that

$$
\log T=1 / \sqrt{\delta}, \quad \log \log \mathrm{T}=\frac{1}{2}|\log \delta|, \quad \mathrm{T}>\mathrm{e}^{2} .
$$

We also set $g(K, d)=\int_{2}^{e^{2}} \frac{\left|E_{K, d}(t)\right|}{t} d t$. Then

$$
\begin{aligned}
& \left|\delta \int_{2}^{\infty} \frac{\mathrm{E}_{\mathrm{K}, \mathrm{d}}(\mathrm{t})}{\mathrm{t}^{1+\delta}} \mathrm{dt}\right| \\
& \quad \leq \delta \int_{2}^{\mathrm{e}^{2}} \frac{\left|\mathrm{E}_{\mathrm{K}, \mathrm{d}}(\mathrm{t})\right|}{\mathrm{t}^{1+\delta}} \mathrm{dt}+\delta \int_{\mathrm{e}^{2}}^{\mathrm{T}} \frac{\left|\mathrm{E}_{\mathrm{K}, \mathrm{d}}(\mathrm{t})\right|}{\mathrm{t}^{1+\delta}} \mathrm{dt}+\delta \int_{\mathrm{T}}^{\infty} \frac{\left|\mathrm{E}_{\mathrm{K}, \mathrm{d}}(\mathrm{t})\right|}{\mathrm{t}^{1+\delta}} \mathrm{dt} \\
& \quad \leq \delta \int_{2}^{\mathrm{e}^{2}} \frac{\left|\mathrm{E}_{\mathrm{K}, \mathrm{d}}(\mathrm{t})\right|}{\mathrm{t}} \mathrm{dt}+\delta \frac{\mathrm{e}(\mathrm{K}, \mathrm{d})}{\log \log \left(\mathrm{e}^{2}\right)} \int_{\mathrm{e}^{2}}^{\mathrm{T}} \frac{\mathrm{dt}}{\mathrm{t}^{1+\delta}}+\delta \frac{\mathrm{e}(\mathrm{K}, \mathrm{d})}{\log \log \mathrm{T}} \int_{\mathrm{T}}^{\infty} \frac{\mathrm{dt}}{\mathrm{t}^{1+\delta}} \\
& \quad \leq \delta \mathrm{g}(\mathrm{K}, \mathrm{d})+\delta \frac{\mathrm{e}(\mathrm{K}, \mathrm{d})}{\log 2} \int_{\mathrm{e}^{2}}^{\mathrm{T}} \frac{\mathrm{dt}}{\mathrm{t}}+\delta \frac{\mathrm{e}(\mathrm{K}, \mathrm{d})}{\log \log \mathrm{T}} \frac{1}{\delta \mathrm{T}^{\delta}} \\
& \quad \leq \delta g(\mathrm{~K}, \mathrm{~d})+\delta \frac{\mathrm{e}(\mathrm{K}, \mathrm{d})}{\log 2} \log \mathrm{l}+\frac{\mathrm{e}(\mathrm{K}, \mathrm{d})}{\log \log \mathrm{T}} \\
& \quad \leq \delta \mathrm{K}(\mathrm{K}, \mathrm{d})+2 \delta \mathrm{e}(\mathrm{K}, \mathrm{d}) \log \mathrm{T}+\frac{2 \mathrm{e}(\mathrm{K}, \mathrm{d})}{|\log \delta|} \\
& \quad=\mathrm{g}(\mathrm{K}, \mathrm{d}) \delta+2 \mathrm{e}(\mathrm{K}, \mathrm{d}) \sqrt{\delta}+\frac{2 \mathrm{e}(\mathrm{K}, \mathrm{d})}{|\log \delta|}
\end{aligned}
$$


so that

$$
\delta \int_{2}^{\infty} \frac{\mathrm{E}_{\mathrm{K}, \mathrm{d}}(\mathrm{t})}{\mathrm{t}^{1+\delta}} \mathrm{dt}=0(\delta), \quad \text { as } \delta \rightarrow 0^{+}
$$

Hence

$$
\begin{aligned}
\sum_{\substack{p \\
K_{p}=K}} \frac{1}{p^{1+\delta}} & =\frac{1}{2 h(d)}(-\gamma-\log \delta+0(\delta))+c(K, d)(1+o(\delta))+o(\delta) \\
& =-\frac{1}{2 h(d)} \log \delta+\left(c(K, d)-\frac{\gamma}{2 h(d)}\right)+o(\delta)
\end{aligned}
$$

as $\delta \rightarrow 0^{+}$. Finally we set $\mathrm{s}=1+\delta$ to obtain the asserted result.

Lemma 5.7 Let $\mathrm{K} \in \mathrm{H}(\mathrm{d})$. Let $\omega$ bea complex number such that $|\omega|=1$.

(i) Theseries

$$
\sum_{\substack{p \\ K_{p}=K}}\left(\sum_{n=2}^{\infty} \frac{\omega^{n}}{n p^{n}}\right)
$$

converges.

(ii) Denoting the sum of the series in (i) by $A(K, d, \omega)$, we have

$$
\sum_{\substack{p \\ K_{p}=K}}\left(\sum_{n=2}^{\infty} \frac{\omega^{n}}{n p^{n s}}\right)=A(K, d, \omega)+o(s-1), \quad \text { ass } \rightarrow 1^{+} .
$$

Proof For $s \geq 1$ wehave

$$
\left|\sum_{n=2}^{\infty} \frac{\omega^{n}}{n p^{n s}}\right| \leq \sum_{n=2}^{\infty} \frac{1}{n p^{n s}} \leq \sum_{n=2}^{\infty} \frac{1}{n p^{n}} \leq \frac{1}{2} \sum_{n=2}^{\infty} \frac{1}{p^{n}}=\frac{1}{2} \frac{1 / p^{2}}{1-1 / p} \leq \frac{1}{p^{2}},
$$

so the series $\sum_{K_{p}=K}\left(\sum_{n=2}^{\infty} \frac{\omega^{n}}{n p^{n s}}\right)$ is uniformly convergent for $s \geq 1$. Thus, in particular, $\sum_{K_{p}=K}\left(\sum_{n=2}^{\infty} \frac{\omega^{n}}{n p^{n}}\right)$ converges, proving (i). M oreover, the uniform convergence ensures that

$$
\lim _{s \rightarrow 1^{+}} \sum_{K_{p}=K}\left(\sum_{n=2}^{\infty} \frac{\omega^{n}}{n p^{n s}}\right)=\sum_{\substack{p \\ K_{p}=K}}\left(\sum_{n=2}^{\infty} \frac{\omega^{n}}{n p^{n}}\right)=A(K, d, \omega),
$$

proving (ii). We note that $\overline{A(K, d, \omega)}=A(K, d, \bar{\omega})$.

Lemma 5.8 Let $K \in H(d)$. Let $\omega$ be a complex number with $|\omega|=1$. Then there exists a nonzero complex number $\mathrm{B}(\mathrm{K}, \mathrm{d}, \omega)$ depending only on $\mathrm{K}, \mathrm{d}$ and $\omega$ such that

$$
\prod_{\substack{p \\ K_{p}=K}}\left(1-\frac{\omega}{p^{s}}\right)=(s-1)^{2 h^{2(d)}} B(K, d, \omega)(1+o(s-1)), \quad \text { as } s \rightarrow 1^{+} .
$$


Proof Let $s$ be a real number with $s>1$. We have as $\left|\omega / p^{s}\right|<1$

$$
\begin{aligned}
& \prod_{\substack{p \\
K_{p}=K}}\left(1-\frac{\omega}{p^{s}}\right)=\prod_{\substack{p \\
K_{p}=K}} e^{\log \left(1-\frac{\omega}{p^{s}}\right)} \\
& =\mathrm{e}^{\sum_{\mathrm{p}}^{K_{\mathrm{p}}=K}} \\
& =e^{-\sum_{k_{p}^{p}=K} \sum_{n=1}^{\infty} \frac{\omega^{n}}{1 p^{15}}} \\
& =e^{-\omega \sum_{\substack{p \\
p_{p}=K}} \frac{1}{p^{s}}-\sum_{K_{p}^{p}=K} \sum_{n=2}^{\infty} \frac{\omega^{n}}{p^{15}}} \\
& =\mathrm{e}^{-\omega\left(-\frac{1}{2 n(d)} \log (s-1)+c(K, d)-\frac{\gamma}{2 \hbar(d)}+0(s-1)\right)-(A(K, d, \omega)+0(s-1))} \\
& \text { (by Lemmas } 5.6 \text { and 5.7(ii)) } \\
& =(s-1)^{\omega / 2 h(d)} B(K, d, \omega)(1+o(s-1)), \quad \text { as } s \rightarrow 1^{+},
\end{aligned}
$$

where

$$
B(K, d, \omega):=e^{\omega\left(\frac{\gamma}{2 n(d)}-c(K, d)\right)-A(K, d, \omega)} \neq 0 .
$$

We note that $\overline{B(K, d, \omega)}=B(K, d, \bar{\omega})$.

Proof of Proposition 5.1 Let $K \in H(d)$. If $p$ is a prime with $K_{p}=K$ then $\left(\frac{d}{p}\right)=0$ or 1 . Hence

$$
\begin{aligned}
& \prod_{\substack{p \\
\left(\frac{d}{p}\right)=1 \\
k_{p}=K}}\left(1-\frac{\omega}{p^{s}}\right)=\frac{\prod_{p}\left(1-\frac{\omega}{k_{p}^{s}}\right)=K}{\prod_{\substack{p \\
\left(\frac{d}{p}\right)=0 \\
k_{p}=K}}\left(1-\frac{\omega}{p^{s}}\right)} \\
& =\frac{(s-1)^{\omega / 2 h(d)} B(K, d, \omega)(1+o(s-1))}{\prod_{\substack{p \\
\left(\frac{d}{p}\right)=0 \\
K_{p}=K}}\left(1-\frac{\omega}{p}\right)(1+o(s-1))} \text { (by Lemma 5.8) } \\
& =(s-1)^{2 h^{\omega}(d)} \mathrm{C}(\mathrm{K}, \mathrm{d}, \omega)(1+0(s-1)), \quad \text { as } s \rightarrow 1^{+},
\end{aligned}
$$

where

$$
C(K, d, \omega):=\frac{B(K, d, \omega)}{\prod_{\substack{p \\\left(\frac{d}{p}\right)=0 \\ K_{p}=K}}\left(1-\frac{\omega}{p}\right)} \neq 0 .
$$

We note that $\overline{\mathrm{C}(\mathrm{K}, \mathrm{d}, \omega)}=\mathrm{C}(\mathrm{K}, \mathrm{d}, \bar{\omega})$. 


\section{The Q uantity $j(K, d)$}

In this section we make use of Proposition 5.1 to determine the limiting behaviour of the infinite product

$$
\prod_{\substack{p \\\left(\frac{p}{p}\right)=1}}\left(1-\frac{f\left(K, K_{p}\right)}{p^{s}}\right)\left(1-\frac{f\left(K, K_{p}\right)^{-1}}{p^{s}}\right)
$$

as $\mathrm{s} \rightarrow 1^{+}$for $\mathrm{K}(\neq \mathrm{I}) \in \mathrm{H}(\mathrm{d})$. We prove

Proposition 6.1 If $K(\neq I) \in H(d)$ then

$$
\lim _{s \rightarrow 1^{+}} \prod_{\substack{p \\\left(\frac{d}{p}\right)=1}}\left(1-\frac{f\left(K, K_{p}\right)}{p^{s}}\right)\left(1-\frac{f\left(K, K_{p}\right)^{-1}}{p^{s}}\right)
$$

exists and is a nonzero real number which we denote by $j(K, d)$.

Proof Let $s$ bea real number with $s>1$. Then

$$
\begin{aligned}
& \prod_{\substack{p \\
\left(\frac{d}{p}\right)=1}}\left(1-\frac{f\left(K, K_{p}\right)}{p^{S}}\right)\left(1-\frac{f\left(K, K_{p}\right)^{-1}}{p^{S}}\right) \\
= & \prod_{\substack{p \\
\left(\frac{d}{p}\right)=1}}\left(1-\frac{e^{2 \pi i\left[K, K_{p}\right]}}{p^{S}}\right)\left(1-\frac{e^{-2 \pi i\left[K, K_{p}\right]}}{p^{S}}\right) \quad(b y(2.21)) \\
= & \prod_{\substack{p \\
\left(\frac{d}{p}\right)=1}}\left(1-\frac{e^{2 \pi i \sum_{j=1}^{\ell} \operatorname{ind}_{A_{j}}(K) \operatorname{ind}_{A_{j}}\left(K_{p}\right) / h_{j}}}{p^{S}}\right)\left(1-\frac{e^{-2 \pi i \sum_{j=1}^{\ell} \operatorname{ind}_{A_{j}}(K) \operatorname{ind}_{A_{j}}\left(K_{p}\right) / h_{j}}}{p^{S}}\right)
\end{aligned}
$$

(by $(2.20)$ )

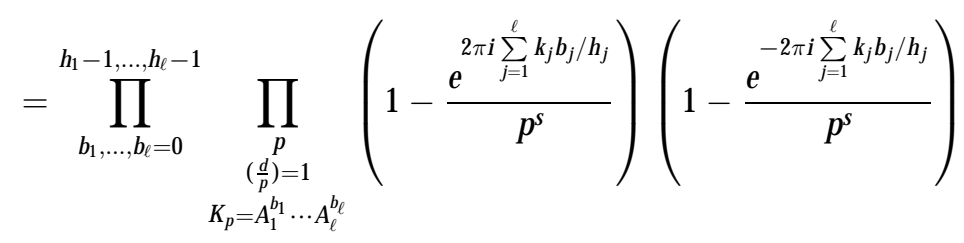

(by (2.19))

$$
=\prod_{b_{1}, \ldots, b_{\ell}=0}^{h_{1}-1, \ldots, h_{\ell}-1}(s-1)^{\frac{1}{2 h(d)} \exp \left(2 \pi i \sum_{j=1}^{\ell} k_{j} b_{j} / h_{j}\right)} C\left(A_{1}^{b_{1}} \cdots A_{\ell}^{b_{\ell}}, d, \exp \left(2 \pi i \sum_{j=1}^{\ell} k_{j} b_{j} / h_{j}\right)\right)
$$




$$
\begin{aligned}
& \times(s-1)^{\frac{1}{2 n(d)} \exp \left(-2 \pi i \sum_{j=1}^{\ell} k_{j} b_{j} / h_{j}\right)} C\left(A_{1}^{b_{1}} \cdots A_{\ell}^{b_{\ell}}, d, \exp \left(-2 \pi i \sum_{j=1}^{\ell} k_{j} b_{j} / h_{j}\right)\right) \\
& \times(1+o(s-1)) \quad(\text { by Proposition 5.1) } \\
& =(s-1)^{\frac{1}{2 \pi(d)}\left(\prod_{j=1}^{\ell}\left(\sum_{b_{j}=0}^{h_{j}-1} \exp \left(2 \pi k_{j} b_{j} / h_{j}\right)\right)+\prod_{j=1}^{\ell}\left(\sum_{b j=0}^{h_{j}-1} \exp \left(-2 \pi i k_{j} b_{j} / h_{j}\right)\right)\right)} \\
& \times \prod_{b_{1}, \cdots, b_{\ell}=0}^{h_{1}-1, \ldots, h_{\ell}-1} C\left(A_{1}^{b_{1}} \cdots A_{\ell}^{b_{\ell}}, d, \exp \left(2 \pi i \sum_{j=1}^{\ell} k_{j} b_{j} / h_{j}\right)\right) \\
& \times C\left(A_{1}^{b_{1}} \cdots A_{\ell}^{b_{\ell}}, d, \exp \left(-2 \pi i \sum_{j=1}^{\ell} k_{j} b_{j} / h_{j}\right)\right)(1+o(s-1)) .
\end{aligned}
$$

As $\mathrm{K} \neq \mathrm{I}$ at least one of $\mathrm{k}_{1}, \ldots, \mathrm{k}_{\ell}$ is nonzero, say $\mathrm{k}_{\mathrm{j}}$, in which case $0<\mathrm{k}_{\mathrm{j}}<\mathrm{h}_{\mathrm{j}}$ and

$$
\sum_{b_{j}=0}^{h_{j}-1} \exp \left( \pm 2 \pi i k_{j} b_{j} / h_{j}\right)=0 .
$$

Thus

$$
\begin{aligned}
\prod_{\substack{p \\
\left(\frac{d}{p}\right)=1}}\left(1-\frac{f\left(K, K_{p}\right)}{p^{s}}\right)\left(1-\frac{f\left(K, K_{p}\right)^{-1}}{p^{s}}\right) \\
=\prod_{b_{1}, \ldots, b_{\ell}=0}^{h_{1}-1, \ldots, h_{\ell}-1} C\left(A_{1}^{b_{1}} \cdots A_{\ell}^{b_{\ell}}, d, \exp \left(2 \pi i \sum_{j=1}^{\ell} k_{j} b_{j} / h_{j}\right)\right) \\
\quad \times C\left(A_{1}^{b_{1}} \cdots A_{\ell}^{b_{\ell}}, d, \exp \left(-2 \pi i \sum_{j=1}^{\ell} k_{j} b_{j} / h_{j}\right)\right) \times(1+o(s-1)) \\
=\prod_{L \in H(d)} C(L, d, f(K, L)) C\left(L, d, f(K, L)^{-1}\right)(1+o(s-1))
\end{aligned}
$$

as $\rightarrow 1^{+}$, by (2.18)-(2.21). Hence

$$
\lim _{s \rightarrow 1^{+}} \prod_{\substack{p \\\left(\frac{d}{p}\right)=1}}\left(1-\frac{f\left(K, K_{p}\right)}{p^{s}}\right)\left(1-\frac{f\left(K, K_{p}\right)^{-1}}{p^{s}}\right)
$$

exists and is equal to

$$
j(K, d):=\prod_{L \in H(d)} C(L, d, f(K, L)) C\left(L, d, f(K, L)^{-1}\right) .
$$


Since each $C\left(L, d, f(K, L)^{ \pm 1}\right)(L \in H(d))$ is a nonzero complex number and

$$
C\left(L, d, f(K, L)^{-1}\right)=C(L, d, \overline{f(K, L)})=\overline{C(L, d, f(K, L))},
$$

we see that $j(K, d)$ is a nonzero real number.

As $f(K, L)^{-1}=f\left(K^{-1}, L\right)$ we see from (6.1) that

$$
j(K, d)=\prod_{L \in H(d)} C(L, d, f(K, L)) C\left(L, d, f\left(K^{-1}, L\right)\right)=j\left(K^{-1}, d\right) .
$$

It is convenient to set

$$
m(K, d):=\frac{t_{1}(d)}{j(K, d)}, \quad K \in H(d),
$$

where $t_{1}(d)$ is defined in (2.32). Thus, appealing to (2.32), Proposition 6.1, and (6.3) we obtain

$$
\begin{aligned}
& m(K, d)=\frac{\prod_{p}\left(1-\frac{1}{p^{2}}\right)}{\lim _{s \rightarrow 1^{+}} \prod_{\substack{p \\
\left(\frac{d}{p}\right)=1}}\left(1-\frac{f\left(K, K_{p}\right)}{p^{s}}\right)\left(1-\frac{f\left(K, K_{p}\right)^{-1}}{p^{s}}\right)} \\
& \lim _{s \rightarrow 1^{+}} \prod_{p}\left(1-\frac{1}{p^{2 s}}\right) \\
& =\frac{\left(\frac{d}{p}\right)=1}{\lim _{s \rightarrow 1^{+}} \prod_{\substack{p \\
\left(\frac{d}{p}\right)=1}}\left(1-\frac{f\left(K, K_{p}\right)}{p^{s}}\right)\left(1-\frac{f\left(K, K_{p}\right)^{-1}}{p^{s}}\right)},
\end{aligned}
$$

that is

$$
m(K, d)=\lim _{s \rightarrow 1^{+}} \prod_{\substack{p \\\left(\frac{d}{p}\right)=1}} \frac{\left(1-\frac{1}{p^{s}}\right)\left(1+\frac{1}{p^{s}}\right)}{\left(1-\frac{f\left(K, K_{p}\right)}{p^{s}}\right)\left(1-\frac{f\left(K, K_{p}\right)^{-1}}{p^{s}}\right)} .
$$

From (6.2) and (6.3) we deduce that

$$
m(K, d)=m\left(K^{-1}, d\right) .
$$

7 Evaluation of $\sum_{n=1}^{\infty} \frac{W_{K}(n)}{n^{s}}, K \neq 1$

In this section we prove the following result.

Proposition 7.1 Let $K(\neq I) \in H(d)$. Then

$$
\sum_{n=1}^{\infty} \frac{W_{K}(n)}{n^{s}}=\ell(K, d) m(K, d)(1+o(s-1)), \quad \text { ass } \rightarrow 1^{+},
$$

where $\ell(K, d)$ is defined in (2.33) and $m(K, d)$ in (6.3). 
Proof Let $K(\neq 1) \in H(d)$. Set $k_{j}=\operatorname{ord}_{A_{j}}(K)(j=1, \ldots, \ell)$, so that $\left(k_{1}, \ldots, k_{\ell}\right) \neq$ $(0, \ldots, 0)$. Let $\mathrm{p}$ bea prime and let $\alpha$ be a positive integer.

First we consider those $p$ for which $\left(\frac{d}{p}\right)=0$ or 1 . Set $b_{j}=\operatorname{ord}_{A_{j}}\left(K_{p}\right)(j=1, \ldots, \ell)$. Let $s_{1}, \ldots, s_{\ell}$ be integers with $0 \leq s_{j} \leq h_{j}-1(j=1, \ldots, \ell)$. Appealing to Definition 2.1, we see that

$$
\begin{aligned}
\mathrm{N}_{\mathrm{A}_{1}^{s_{1}} \ldots \mathrm{A}_{\ell}^{s_{\ell}}\left(\mathrm{p}^{\alpha}\right)} & =\text { number of } \varepsilon(= \pm 1) \text { such that } \mathrm{K}_{\mathrm{p}}^{\alpha \varepsilon}=\mathrm{A}_{1}^{\mathrm{s}_{1}} \cdots \mathrm{A}_{\ell}^{\mathrm{s}_{\ell}} \\
& =\text { number of } \varepsilon(= \pm 1) \text { such that } \mathrm{A}_{1}^{\mathrm{b}_{1} \alpha \varepsilon} \cdots \mathrm{A}_{\ell}^{\mathrm{b}_{\ell} \alpha \varepsilon}=\mathrm{A}_{1}^{\mathrm{s}_{1}} \cdots \mathrm{A}_{\ell}^{\mathrm{s}_{\ell}} \\
& =\text { number of } \varepsilon(= \pm 1) \text { such that } \mathrm{b}_{\mathrm{j}} \alpha \varepsilon \equiv \mathrm{s}_{\mathrm{j}} \quad\left(\bmod \mathrm{h}_{\mathrm{j}}\right)(\mathrm{j}=1, \ldots, \ell)
\end{aligned}
$$

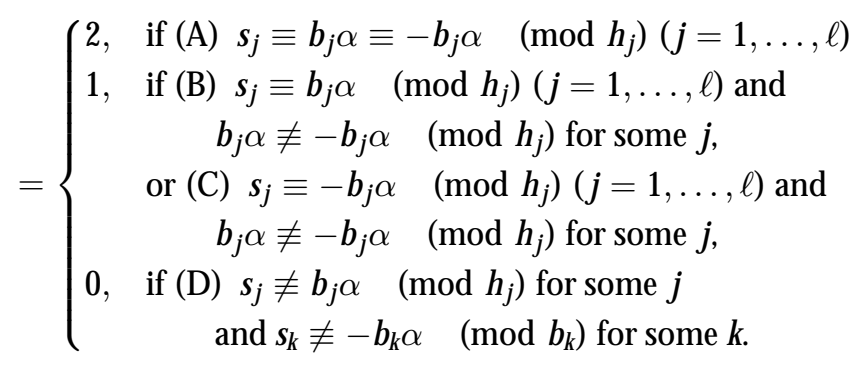

Hence

$$
\begin{aligned}
\sum_{L \in H(d)} f(K, L) N_{L}\left(p^{\alpha}\right) & =\sum_{s_{1}, \ldots, s_{\ell}=0}^{h_{1}-1, \ldots, h_{\ell}-1} e^{2 \pi i \sum_{j=1}^{\ell} k_{j} s_{j} / h_{j}} N_{A_{1}^{s_{1}} \ldots A_{\ell}^{s_{\ell}}}\left(p^{\alpha}\right) \\
& =\left\{2 \sum_{(A)}+\sum_{(B)}+\sum_{(C)}\right\} e^{2 \pi i \sum_{j=1}^{\ell} k_{j} s_{j} / h_{j}} \\
& =\left\{\left(\sum_{(A)}+\sum_{(B)}\right)+\left(\sum_{(A)}+\sum_{(C)}\right)\right\} e^{2 \pi i \sum_{j=1}^{\ell} k_{j} s_{j} / h_{j}} \\
& =\left\{\sum_{(A) \cup(B)}+\sum_{(A) \cup(C)}\right\} e^{2 \pi i \sum_{j=1}^{\ell} k_{j} s_{j} / h_{j}} \\
& =e^{2 \pi i \alpha \sum_{j=1}^{\ell} k_{j} s_{j} / h_{j}}+e^{-2 \pi i \alpha \sum_{j=1}^{\ell} k_{j} s_{j} / h_{j}} \\
& =\theta^{\alpha}+\theta^{-\alpha},
\end{aligned}
$$

where we have set for convenience

$$
\theta:=e^{2 \pi i \sum_{j=1}^{\ell} k_{j} b_{j} / h_{j}}=e^{2 \pi i\left[K, K_{p}\right]}=f\left(K, K_{p}\right) .
$$

Note that $\theta$ depends on $p, d$ and $K$, and that $|\theta|=1$. If $\left(\frac{d}{p}\right)=1$, from Proposition 3.1, we deduce that

$$
P_{L}\left(p^{\alpha}\right)=w(d) N_{L}\left(p^{\alpha}\right) \quad(L \in H(d))
$$


so that

$$
\begin{aligned}
W_{K}\left(p^{\alpha}\right) & =\frac{1}{W(d)} \sum_{L \in H(d)} f(K, L) P_{L}\left(p^{\alpha}\right) \\
& =\sum_{L \in H(d)} f(K, L) N_{L}\left(p^{\alpha}\right) \\
& =\theta^{\alpha}+\theta^{-\alpha} .
\end{aligned}
$$

Thus

$$
\begin{aligned}
\sum_{\alpha=1}^{\infty} \frac{W_{k}\left(\mathrm{p}^{\alpha}\right)}{\mathrm{p}^{\alpha s}} & =\sum_{\alpha=1}^{\infty} \frac{\theta^{\alpha}+\theta^{-\alpha}}{\mathrm{p}^{\alpha s}} \\
& =\frac{\theta / \mathrm{p}^{s}}{1-\theta / \mathrm{p}^{s}}+\frac{\theta^{-1} / \mathrm{p}^{s}}{1-\theta^{-1} / \mathrm{p}^{s}} \\
& =\frac{\theta / \mathrm{p}^{s}+\theta^{-1} / \mathrm{p}^{s}-2 / \mathrm{p}^{2 s}}{\left(1-\theta / \mathrm{p}^{s}\right)\left(1-\theta^{-1} / \mathrm{p}^{s}\right)}
\end{aligned}
$$

so that by (2.24)

$$
\sum_{j=0}^{\infty} \frac{W_{k}\left(p^{j}\right)}{p^{j s}}=1+\frac{\theta / p^{s}+\theta^{-1} / p^{s}-2 / p^{2 s}}{\left(1-\theta / p^{s}\right)\left(1-\theta^{-1} / p^{s}\right)}
$$

that is

$$
\sum_{j=0}^{\infty} \frac{W_{k}\left(p^{j}\right)}{p^{j s}}=\frac{1-1 / p^{2 s}}{\left(1-\theta / p^{s}\right)\left(1-\theta^{-1} / p^{s}\right)}, \quad \text { if }\left(\frac{d}{p}\right)=1
$$

If $\left(\frac{d}{p}\right)=0$ then, by Proposition 3.1, we have

$$
P_{\mathrm{L}}\left(p^{\alpha}\right)= \begin{cases}0, & \text { if } \alpha \geq 2, \\ \frac{w(d)}{2} N_{\mathrm{L}}(p), & \text { if } \alpha=1 .\end{cases}
$$

Hence

$$
\begin{aligned}
W_{K}\left(p^{\alpha}\right) & =\frac{1}{W(d)} \sum_{L \in H(d)} f(K, L) P_{L}\left(p^{\alpha}\right) \\
& = \begin{cases}0, & \text { if } \alpha \geq 2, \\
\frac{1}{2} \sum_{L \in H(d)} f(K, L) N_{L}(p), & \text { if } \alpha=1,\end{cases} \\
& = \begin{cases}0, & \text { if } \alpha \geq 2, \\
\frac{1}{2}\left(\theta+\theta^{-1}\right), & \text { if } \alpha=1 .\end{cases}
\end{aligned}
$$


Thus

$$
\sum_{j=0}^{\infty} \frac{W_{k}\left(p^{j}\right)}{p^{j s}}=1+\frac{\frac{1}{2}\left(\theta+\theta^{-1}\right)}{p^{s}}, \quad \text { if }\left(\frac{d}{p}\right)=0 .
$$

Now suppose that $\left(\frac{d}{p}\right)=-1$. By Proposition 3.1 we have $P_{L}\left(p^{\alpha}\right)=0$. Then, by (2.23), we deduce that $\mathrm{W}_{\mathrm{K}}\left(\mathrm{p}^{\alpha}\right)=0$. Thus

$$
\sum_{j=0}^{\infty} \frac{W_{k}\left(p^{j}\right)}{p^{j s}}=1, \quad \text { if }\left(\frac{d}{p}\right)=-1
$$

Next, by Proposition 4.1, $W_{K}(n)$ is a multiplicative function of $n$ so that

$$
\sum_{n=1}^{\infty} \frac{W_{k}(n)}{n^{s}}=\prod_{p}\left(\sum_{j=0}^{\infty} \frac{W_{k}\left(p^{j}\right)}{p^{j s}}\right)
$$

Appealing to (7.1), (7.2) and (7.3), we obtain

$$
\sum_{n=1}^{\infty} \frac{W_{K}(n)}{n^{s}}=\prod_{\substack{p \\\left(\frac{d}{p}\right)=1}} \frac{\left(1-\frac{1}{p^{2 s}}\right)}{\left(1-\frac{\theta}{p^{s}}\right)\left(1-\frac{\theta^{-1}}{p^{s}}\right)} \prod_{\substack{p \\\left(\frac{d}{p}\right)=0}}\left(1+\frac{\frac{1}{2}\left(\theta+\theta^{-1}\right)}{p^{s}}\right) .
$$

We now consider the product

$$
\begin{aligned}
\prod_{\substack{p \\
\left(\frac{d}{p}\right)=1}}\left(1-\frac{\theta}{p^{s}}\right)\left(1-\frac{\theta^{-1}}{p^{s}}\right) & =\prod_{\substack{p \\
\left(\frac{d}{p}\right)=1}}\left(1-\frac{f\left(K, K_{p}\right)}{p^{s}}\right)\left(1-\frac{f\left(K, K_{p}\right)^{-1}}{p^{s}}\right) \\
& =j(K, d)(1+o(s-1)), \quad \text { as } s \rightarrow 1^{+},
\end{aligned}
$$

by Proposition 6.1. Herewe used $\mathrm{K} \neq \mathrm{I}$.

Further

$$
\prod_{\substack{p \\\left(\frac{d}{p}\right)=1}}\left(1-\frac{1}{p^{2 s}}\right)=t_{1}(d)(1+o(s-1)), \quad \text { as } \rightarrow 1^{+},
$$


where $_{1}(d)$ is defined in (2.32). Also

$$
\begin{aligned}
\prod_{\substack{p \\
\left(\frac{d}{p}\right)=0}}\left(1+\frac{\frac{1}{2}\left(\theta+\theta^{-1}\right)}{p^{s}}\right) & =\prod_{\substack{p \\
\left(\frac{d}{p}\right)=0}}\left(1+\frac{\frac{1}{2}\left(f\left(K, K_{p}\right)+f\left(K, K_{p}\right)^{-1}\right)}{p^{s}}\right) \\
& =\prod_{\substack{p \\
\left(\frac{d}{p}\right)=0}}\left(1+\frac{\frac{1}{2}\left(f\left(K, K_{p}\right)+f\left(K, K_{p}\right)^{-1}\right)}{p}\right)(1+o(s-1)) \\
& =\prod_{\substack{p \\
\left(\frac{d}{p}\right)=0}}\left(1+\frac{f\left(K, K_{p}\right)}{p}\right)(1+o(s-1)) \\
& =\ell(K, d)(1+o(s-1)), \text { as } \rightarrow 1^{+},
\end{aligned}
$$

since

$$
\left(\frac{d}{p}\right)=0 \Rightarrow K_{p}=K_{p}^{-1} \Rightarrow f\left(K, K_{p}\right)^{-1}=f\left(K, K_{p}^{-1}\right)=f\left(K, K_{p}\right) .
$$

We have shown that

$$
\begin{aligned}
\sum_{n=1}^{\infty} \frac{W_{K}(n)}{n^{s}} & =\frac{t_{1}(d)(1+o(s-1)) \ell(K, d)(1+o(s-1))}{j(K, d)(1+o(s-1))} \\
& =\ell(K, d) m(K, d)(1+o(s-1)), \quad \text { as } s \rightarrow 1^{+}
\end{aligned}
$$

The next result is a slight modification of Proposition 7.1, in a form convenient for use in the proof of Proposition 9.1.

Proposition 7.2 Let $K(\neq I) \in H(d)$. Then

$$
\zeta(2 s) \sum_{n=1}^{\infty} \frac{W_{K}(n)}{n^{s}}=\frac{\pi^{2}}{6} \ell(K, d) m(K, d)(1+o(s-1)), \quad \text { as } s \rightarrow 1^{+} .
$$

Proof By Proposition 7.1 we have

$$
\sum_{n=1}^{\infty} \frac{W_{K}(n)}{n^{s}}=\ell(K, d) m(K, d)(1+o(s-1)), \quad \text { as } s \rightarrow 1^{+} .
$$

Also

$$
\zeta(2 s)=\zeta(2)(1+0(s-1))=\frac{\pi^{2}}{6}(1+0(s-1)), \quad \text { as } s \rightarrow 1^{+} .
$$

Hence

$$
\zeta(2 s) \sum_{n=1}^{\infty} \frac{W_{K}(n)}{n^{s}}=\frac{\pi^{2}}{6} \ell(K, d) m(K, d)(1+o(s-1)), \quad \text { as } s \rightarrow 1^{+} .
$$


8 Evaluation of $\sum_{n=1}^{\infty} \frac{W_{1}(n)}{n^{s}}$

In this section we determine $\zeta(2 s) \sum_{n=1}^{\infty} \frac{W_{k}(n)}{n^{s}}$ as $s \rightarrow 1^{+}$in the excluded case $K=1$. This is the companion result to Proposition 7.2.

\section{Proposition 8.1}

$$
\begin{aligned}
\zeta(2 s) \sum_{n=1}^{\infty} \frac{W_{1}(n)}{n^{s}} \\
=\frac{2 \pi h(d)}{w(d) \sqrt{|d|} \frac{1}{s-1}} \\
+\left\{\frac{4 \pi \gamma h(d)}{w(d) \sqrt{|d|}}+\frac{2 \pi(\log 2 \pi) h(d)}{w(d) \sqrt{|d|}}-\frac{\pi}{\sqrt{|d|}} \sum_{m=1}^{|d|}\left(\frac{d}{m}\right) \log \Gamma\left(\frac{m}{|d|}\right)\right\} \\
+o(s-1),
\end{aligned}
$$

ass $\rightarrow 1^{+}$.

Proof From (2.25) wehave

$$
W_{I}(n)=\frac{P(n)}{W(d)}
$$

so that

$$
\sum_{n=1}^{\infty} \frac{W_{1}(n)}{n^{s}}=\frac{1}{W(d)} \sum_{n=1}^{\infty} \frac{P(n)}{n^{s}}, \quad s>1
$$

Thus

$$
\begin{aligned}
\zeta(2 s) \sum_{n=1}^{\infty} \frac{W_{1}(n)}{n^{s}} & =\frac{1}{w(d)} \sum_{m=1}^{\infty} \frac{1}{m^{2 s}} \sum_{n=1}^{\infty} \frac{P(n)}{n^{s}} \\
& =\frac{1}{w(d)} \sum_{\ell=1}^{\infty} \frac{1}{\ell^{s}} \sum_{\substack{m, n \\
m^{2} n=\ell}} P(n) \\
& =\frac{1}{w(d)} \sum_{\ell=1}^{\infty} \frac{1}{\ell^{s}} \sum_{m^{2} \mid \ell} P\left(\ell / m^{2}\right) \\
& =\frac{1}{w(d)} \sum_{\ell=1}^{\infty} \frac{R(\ell)}{\ell^{s}},
\end{aligned}
$$

by (2.7). Now, by Dirichlet's formula (see for example [5], [6]), we have

$$
R(\ell)=w(d) \sum_{e \mid \ell}\left(\frac{d}{e}\right),
$$


so that

$$
\begin{aligned}
\zeta(2 \mathrm{~s}) \sum_{\mathrm{n}=1}^{\infty} \frac{\mathrm{W}_{\mathrm{I}}(\mathrm{n})}{\mathrm{n}^{\mathrm{s}}} & =\sum_{\ell=1}^{\infty} \frac{1}{\ell^{\mathrm{s}}} \sum_{\mathrm{e} \mid \ell}\left(\frac{\mathrm{d}}{\mathrm{e}}\right) \\
& =\sum_{\mathrm{e}, \mathrm{k}=1}^{\infty} \frac{1}{\mathrm{e}^{\mathrm{s} k^{s}}}\left(\frac{\mathrm{d}}{\mathrm{e}}\right) \\
& =\left(\sum_{\mathrm{e}=1}^{\infty} \frac{\left(\frac{\mathrm{d}}{\mathrm{e}}\right)}{\mathrm{e}^{\mathrm{s}}}\right)\left(\sum_{\mathrm{k}=1}^{\infty} \frac{1}{\mathrm{k}^{s}}\right) \\
& =L(\mathrm{~s}, \mathrm{~d}) \zeta(\mathrm{s}) .
\end{aligned}
$$

Ass $\rightarrow 1^{+}$we have

$$
\zeta(\mathrm{s})=\frac{1}{\mathrm{~s}-1}+\gamma+0(\mathrm{~s}-1),
$$

where $\gamma$ denotes Euler's constant, and

$$
L(s, d)=L(1, d)+(s-1) L^{\prime}(1, d)+o\left((s-1)^{2}\right),
$$

so that

$$
\zeta(2 s) \sum_{n=1}^{\infty} \frac{W_{1}(n)}{n^{s}}=\frac{L(1, d)}{s-1}+\left(\gamma L(1, d)+L^{\prime}(1, d)\right)+o(s-1) .
$$

Now, from [1, p. 171] and [8, p. 110], we have

$$
L(1, d)=\frac{2 \pi h(d)}{w(d) \sqrt{|d|}}
$$

and

$$
L^{\prime}(1, d)=\frac{2 h(d) \pi(\gamma+\log 2 \pi)}{w(d) \sqrt{|d|}}-\frac{\pi}{\sqrt{|d|}} \sum_{m=1}^{|d|}\left(\frac{d}{m}\right) \log \Gamma\left(\frac{m}{|d|}\right)
$$

so that

$$
\begin{aligned}
\zeta(2 s) \sum_{n=1}^{\infty} \frac{W_{1}(n)}{n^{s}} \\
=\frac{2 \pi h(d)}{w(d) \sqrt{|d|}} \frac{1}{s-1} \\
\quad+\left\{\frac{4 \pi \gamma h(d)}{w(d) \sqrt{|d|}}+\frac{2 \pi(\log 2 \pi) h(d)}{w(d) \sqrt{|d|}}-\frac{\pi}{\sqrt{|d|}} \sum_{m=1}^{|d|}\left(\frac{d}{m}\right) \log \Gamma\left(\frac{m}{|d|}\right)\right\} \\
+o(s-1), \quad \text { as } s \rightarrow 1^{+},
\end{aligned}
$$

as asserted. 


\section{Evaluation of $|\eta((b+\sqrt{d}) / 2 a)|$}

Let $K \in H(d)$. In this section we determine $\sum_{n=1}^{\infty} \frac{R_{K}(n)}{n^{s}}(s>1)$ in two different ways (Propositions 9.1 and 9.2). First we make use of Propositions 7.2 and 8.1 to prove Proposition 9.1. Secondly we appeal to Kronecker's limit formula to prove Proposition 9.2. Equating the expressions in Propositions 9.1 and 9.2, we obtain the main result of this paper which gives a formula for $|\eta((b+\sqrt{d}) / 2 a)|$ (Theorem 9.3).

Proposition 9.1 Let $K \in H(d)$. Then

$$
\sum_{n=1}^{\infty} \frac{R_{K}(n)}{n^{s}}=\frac{2 \pi / \sqrt{|d|}}{s-1}+A(K, d)+0(s-1), \quad \text { ass } \rightarrow 1^{+}
$$

with

$$
\begin{gathered}
A(K, d)=\frac{4 \pi \gamma}{\sqrt{|d|}}+\frac{2 \pi(\log 2 \pi)}{\sqrt{|d|}}-\frac{\pi w(d)}{h(d) \sqrt{|d|}} \sum_{m=1}^{|d|}\left(\frac{d}{m}\right) \log \Gamma\left(\frac{m}{|d|}\right) \\
+\frac{\pi^{2} w(d)}{6 h(d)} \sum_{\substack{L \in H(d) \\
L \neq 1}} f(L, K) \ell(L, d) m(L, d),
\end{gathered}
$$

where $f(L, K)$ is defined in (2.21), $\ell(L, d)$ in (2.33), and $m(L, d)$ in (6.3).

Proof Appealing to (2.22) and (2.23), we obtain

$$
\begin{aligned}
\sum_{L \in H(d)} f(L, K)^{-1} W_{L}(n) & =\frac{1}{w(d)} \sum_{L \in H(d)} f(L, K)^{-1} \sum_{M \in H(d)} f(L, M) P_{M}(n) \\
& =\frac{1}{w(d)} \sum_{M \in H(d)} P_{M}(n) \sum_{L \in H(d)} f(M, L) f(L, K)^{-1} \\
& =\frac{1}{w(d)} \sum_{\substack{M \in H(d) \\
M=K}} P_{M}(n) h(d) \\
& =\frac{h(d)}{w(d)} P_{K}(n)
\end{aligned}
$$

so that

$$
P_{K}(n)=\frac{w(d)}{h(d)} \sum_{L \in H(d)} f(L, K)^{-1} W_{L}(n)
$$

Hence

$$
\sum_{n=1}^{\infty} \frac{P_{K}(n)}{n^{s}}=\frac{W(d)}{h(d)} \sum_{L \in H(d)} f(L, K)^{-1} \sum_{n=1}^{\infty} \frac{W_{L}(n)}{n^{s}}
$$


By (2.5) we have

$$
\sum_{n=1}^{\infty} \frac{R_{k}(n)}{n^{s}}=\zeta(2 s) \sum_{n=1}^{\infty} \frac{P_{k}(n)}{n^{s}}
$$

so that

$$
\sum_{n=1}^{\infty} \frac{R_{K}(n)}{n^{s}}=\frac{w(d)}{h(d)} \sum_{L \in H(d)} f(L, K)^{-1}\left(\zeta(2 s) \sum_{n=1}^{\infty} \frac{W_{L}(n)}{n^{s}}\right) .
$$

Appealing to Proposition 7.2 and 8.1 , we obtain as $\mathrm{f}(\mathrm{I}, \mathrm{K})=1$

$$
\begin{aligned}
& \sum_{n=1}^{\infty} \frac{R_{K}(n)}{n^{s}}=\frac{w(d)}{h(d)}\left\{\frac{2 \pi h(d)}{w(d) \sqrt{|d|}} \frac{1}{s-1}+\frac{4 \pi \gamma h(d)}{w(d) \sqrt{|d|}}\right.+\frac{2 \pi(\log 2 \pi) h(d)}{w(d) \sqrt{|d|}}-\frac{\pi}{\sqrt{|d|}} \sum_{m=1}^{|d|}\left(\frac{d}{m}\right) \log \Gamma\left(\frac{m}{|d|}\right)+o(s-1) \\
&\left.+\sum_{\substack{L \in H(d) \\
L \neq 1}} f(L, K)^{-1} \frac{\pi^{2}}{6} \ell(L, d) m(L, d)(1+0(s-1))\right\} \\
&=\frac{2 \pi / \sqrt{|d|}}{s-1}+\left\{\frac{4 \pi \gamma}{\sqrt{|d|}}+\frac{2 \pi(\log 2 \pi)}{\sqrt{|d|}}-\frac{\pi w(d)}{h(d) \sqrt{|d|}} \sum_{m=1}^{|d|}\left(\frac{d}{m}\right) \log \Gamma\left(\frac{m}{|d|}\right)\right. \\
&\left.\quad+\frac{\pi^{2}}{6} \frac{w(d)}{h(d)} \sum_{\substack{L \in H(d) \\
L \neq 1}} f(L, K)^{-1} \ell(L, d) m(L, d)\right\}+o(s-1)
\end{aligned}
$$

as $\mathrm{s} \rightarrow 1^{+}$. The asserted formula now follows as

$$
\begin{aligned}
\sum_{\substack{L \in H(d) \\
L \neq 1}} f(L, K)^{-1} \ell(L, d) m(L, d) & =\sum_{\substack{L \in H(d) \\
L \neq 1}} f\left(L^{-1}, K\right) \ell(L, d) m(L, d) \\
& =\sum_{\substack{L \in H(d) \\
L \neq 1}} f(L, K) \ell\left(L^{-1}, d\right) m\left(L^{-1}, d\right) \quad\left(L \rightarrow L^{-1}\right) \\
& =\sum_{\substack{L \in H(d) \\
L \neq 1}} f(L, K) \ell(L, d) m(L, d)
\end{aligned}
$$

by (2.34) and (6.5).

Proposition 9.2 Let $K=[a, b, c] \in H(d)$. Then

$$
\sum_{n=1}^{\infty} \frac{R_{k}(n)}{n^{s}}=\frac{2 \pi / \sqrt{|d|}}{s-1}+B(a, b, c)+o(s-1), \quad \text { ass } \rightarrow 1^{+},
$$


where

$$
B(a, b, c)=\frac{4 \pi \gamma}{\sqrt{|d|}}-\frac{2 \pi \log |d|}{\sqrt{|d|}}-\frac{8 \pi}{\sqrt{|d|}} \log \left(a^{-1 / 4}\left|\eta\left(\frac{b+\sqrt{d}}{2 a}\right)\right|\right) .
$$

Proof Wehave for $n \geq 1$

$$
R_{K}(n)=R_{(a, b, c)}(n)=\sum_{\substack{x, y=-\infty \\
a x^{2}+b x y+c y^{2}=n}}^{\infty} 1=\sum_{\begin{array}{c}
x, y=-\infty \\
(x, y) \neq(0,0) \\
a x^{2}+b x y+c y^{2}=n
\end{array}}^{\infty} 1,
$$

so that, by Kronecker's limit formula (see for example [9, Theorem 1, p. 14]), we obtain

$$
\begin{aligned}
\sum_{n=1}^{\infty} \frac{R_{k}(n)}{n^{s}} & =\sum_{n=1}^{\infty} \frac{1}{n^{s}} \sum_{\substack{x, y=-\infty \\
(x, y) \neq(0,0) \\
a x^{2}+b x y+c y^{2}=n}}^{\infty} 1 \\
& =\sum_{\substack{x, y=-\infty \\
(x, y) \neq(0,0)}}^{\infty} \frac{1}{\left(a x^{2}+b x y+c y^{2}\right)^{s}} \\
& =\frac{2 \pi / \sqrt{|d|}}{s-1}+B(a, b, c)+o(s-1),
\end{aligned}
$$

as $\rightarrow 1^{+}$, where

$$
\mathrm{B}(\mathrm{a}, \mathrm{b}, \mathrm{c})=\frac{4 \pi \gamma}{\sqrt{|\mathrm{d}|}}-\frac{2 \pi \log |\mathrm{d}|}{\sqrt{|\mathrm{d}|}}-\frac{8 \pi}{\sqrt{|\mathrm{d}|}} \log \left(\mathrm{a}^{-1 / 4}\left|\eta\left(\frac{\mathrm{b}+\sqrt{\mathrm{d}}}{2 \mathrm{a}}\right)\right|\right) .
$$

Theorem 9.3 Let $K=[a, b, c] \in H(d)$. Then

$$
\begin{aligned}
& a^{-1 / 4}\left|\eta\left(\frac{b+\sqrt{d}}{2 a}\right)\right| \\
& =(2 \pi|d|)^{-1 / 4}\left\{\prod_{m=1}^{|d|} \Gamma\left(\frac{m}{|d|}\right)^{\left(\frac{d}{m}\right)}\right\}^{\frac{w(d)}{8 h(d)}} e^{-\frac{\pi w(d) \sqrt{|d|}}{48 h(d)}} \sum_{\substack{L \in H(d) \\
L \neq 1}} f(L, K) \ell(L, d) m(L, d)
\end{aligned}
$$

where $f(L, K)$ is defined in (2.21), $\ell(L, d)$ in (2.33), and $m(L, d)$ in (6.3).

Proof From Propositions 9.1 and 9.2, we deduce that

$$
A(K, d)=B(a, b, c)
$$


so that

$$
\begin{gathered}
\frac{4 \pi \gamma}{\sqrt{|d|}+} \frac{2 \pi(\log 2 \pi)}{\sqrt{|d|}}-\frac{\pi w(d)}{h(d) \sqrt{|d|}} \sum_{m=1}^{|d|}\left(\frac{d}{m}\right) \log \Gamma\left(\frac{m}{|d|}\right) \\
+\frac{\pi^{2} w(d)}{6 h(d)} \sum_{\substack{L \in H(d) \\
L \neq 1}} f(L, K) \ell(L, d) m(L, d) \\
=\frac{4 \pi \gamma}{\sqrt{|d|}}-\frac{2 \pi \log |d|}{\sqrt{|d|}}-\frac{8 \pi}{\sqrt{|d|}} \log \left(a^{-1 / 4}\left|\eta\left(\frac{b+\sqrt{d}}{2 a}\right)\right|\right) .
\end{gathered}
$$

Multiplying both sides by $\sqrt{|\mathrm{d}|} / 8 \pi$, and rearranging terms, we obtain

$$
\begin{aligned}
\log \left(a^{-1 / 4} \mid \eta\left(\frac{b+\sqrt{d}}{2 a}\right)\right)= & \log \left((2 \pi|d|)^{-1 / 4}\left(\prod_{m=1}^{|d|} \Gamma\left(\frac{m}{|d|}\right)^{\left(\frac{d}{m}\right)}\right)^{\frac{w(d)}{8 h(d)}}\right) \\
& -\frac{\pi w(d) \sqrt{|d|}}{48 h(d)} \sum_{\substack{L \in H(d) \\
L \neq 1}} f(L, K) \ell(L, d) m(L, d) .
\end{aligned}
$$

Exponentiating both sides, we obtain the asserted formula.

We close this section by illustrating Theorem 9.3 in the cases $d=-15$ and $d=-31$. First we treat $d=-15$.

Example $9.4 d=-15$. In this case we have

$$
H(-15)=\{I, A\}, \quad A^{2}=I,
$$

where

$$
\mathrm{I}=[1,1,4], \quad \mathrm{A}=[2,1,2],
$$

so that $h(-15)=2$. The primes dividing $d=-15$ are 3 and 5 . Appealing to formulae (2.11) and (2.12) we obtain

$$
\mathrm{K}_{3}=[3,3,2]=[2,-3,3]=[2,1,2]=\mathrm{A}
$$

and

$$
\mathrm{K}_{5}=[5,5,2]=[2,-5,5]=[2,-1,2]=[2,1,2]=\mathrm{A} .
$$

Next (with $A_{1}=A, h_{1}=2, \ell=1$ ) we have from (2.20)

$$
[I, I]=[I, A]=[A, I]=0, \quad[A, A]=1 / 2,
$$

so that by $(2.21)$

$$
f(I, I)=f(I, A)=f(A, I)=1, \quad f(A, A)=-1 .
$$


Then, by (2.33), we have

$$
\ell(A,-15)=\left(1+\frac{f\left(A, K_{3}\right)}{3}\right)\left(1+\frac{f\left(A, K_{5}\right)}{5}\right)=\left(1-\frac{1}{3}\right)\left(1-\frac{1}{5}\right)=\frac{8}{15} .
$$

Further, by (6.4) we deduce that

$$
m(A,-15)=\lim _{s \rightarrow 1^{+}} \prod_{\substack{p \\\left(\frac{15}{p}\right)=1}} \frac{\left(1-\frac{1}{p^{s}}\right)\left(1+\frac{1}{p^{s}}\right)}{\left(1-\frac{f\left(A, K_{p}\right)}{p^{s}}\right)\left(1-\frac{f\left(A, K_{p}\right)-1}{p^{s}}\right)} .
$$

As $\mathrm{H}(-15)$ is a cyclic group of order 2 , by Gauss' theory of genera, we have for $\mathrm{p} \neq 3,5$

$$
f\left(A, K_{p}\right)=1 \longleftrightarrow K_{p}=I \longleftrightarrow\left(\frac{-3}{p}\right)=\left(\frac{5}{p}\right)=1
$$

and

$$
f\left(A, K_{p}\right)=-1 \longleftrightarrow K_{p}=A \longleftrightarrow\left(\frac{-3}{p}\right)=\left(\frac{5}{p}\right)=-1
$$

so that

$$
m(A,-15)=\lim _{s \rightarrow 1^{+}} \prod_{\substack{p \\\left(\frac{-15}{p}\right)=1}} \frac{\left(1-\frac{1}{p^{s}}\right)\left(1+\frac{1}{p^{s}}\right)}{\left(1-\frac{\left(\frac{-3}{p}\right)}{p^{3}}\right)\left(1-\frac{\left(\frac{5}{p}\right)}{p^{5}}\right)} .
$$

If $p$ is a prime with $\left(\frac{-15}{p}\right)=-1$, we have $\left(\left(\frac{-3}{p}\right),\left(\frac{5}{p}\right)\right)=(1,-1)$ or $(-1,1)$ so

$$
m(A,-15)=\lim _{s \rightarrow 1^{+}} \prod_{p \neq 3,5} \frac{\left(1-\frac{1}{p^{s}}\right)\left(1+\frac{1}{p^{s}}\right)}{\left(1-\frac{\left(\frac{-3}{p}\right)}{p^{s}}\right)\left(1-\frac{\left(\frac{5}{p}\right)}{p^{5}}\right)} .
$$

$\operatorname{As}\left(\frac{-3}{5}\right)=\left(\frac{5}{3}\right)=-1$ we have

$$
\begin{aligned}
\mathrm{m}(\mathrm{A},-15) & =\lim _{s \rightarrow 1^{+}} \frac{1}{\left(1-\frac{1}{3^{5}}\right)\left(1-\frac{1}{5^{s}}\right)} \prod_{\mathrm{p}} \frac{\left(1-\frac{1}{\mathrm{p}^{s}}\right)\left(1+\frac{1}{\mathrm{p}^{s}}\right)}{\left(1-\frac{\left(\frac{-3}{\mathrm{p}}\right)}{\mathrm{p}^{s}}\right)\left(1-\frac{\left(\frac{5}{p}\right)}{\mathrm{p}^{s}}\right)} \\
& =\frac{15}{8} \lim _{\mathrm{s} \rightarrow 1^{+}} \prod_{\mathrm{p}} \frac{\left(1-\frac{\left(\frac{-3}{p}\right)}{\mathrm{p}^{s}}\right)^{-1}\left(1-\frac{\left(\frac{5}{p}\right)}{\mathrm{p}^{s}}\right)}{\left(1-\frac{1}{\mathrm{p}^{25}}\right)^{-1}} \\
& =\frac{15}{8} \lim _{\mathrm{s} \rightarrow 1^{+}} \frac{\mathrm{L}(\mathrm{s},-3) \mathrm{L}(\mathrm{s}, 5)}{\zeta(2 \mathrm{~s})} \\
& =\frac{15}{8} \frac{\mathrm{L}(1,-3) \mathrm{L}(1,5)}{\zeta(2)}
\end{aligned}
$$




$$
\begin{aligned}
& =\frac{15}{8} \frac{\frac{\pi}{3 \sqrt{3}} \frac{2}{\sqrt{5}} \log \left(\frac{1+\sqrt{5}}{2}\right)}{\pi^{2} / 6} \\
& =\frac{\sqrt{15}}{2 \pi} \log \left(\frac{1+\sqrt{5}}{2}\right) .
\end{aligned}
$$

By Theorem 9.3, for $K=[a, b, c] \in H(-15)$, we have

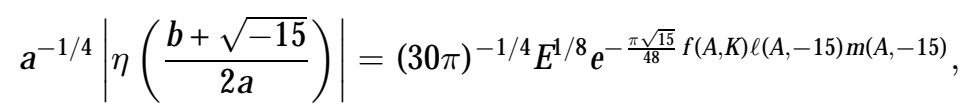

where

$$
E=\frac{\Gamma(1 / 15) \Gamma(2 / 15) \Gamma(4 / 15) \Gamma(8 / 15)}{\Gamma(7 / 15) \Gamma(11 / 15) \Gamma(13 / 15) \Gamma(14 / 15)} .
$$

Taking $(a, b, c)=(1,1,4)$, we obtain

$$
\left|\eta\left(\frac{1+\sqrt{-15}}{2}\right)\right|=(30 \pi)^{-1 / 4} \mathrm{E}^{1 / 8}\left(\frac{1+\sqrt{5}}{2}\right)^{-1 / 12},
$$

and with $(a, b, c)=(2,1,2)$

$$
2^{-1 / 4}\left|\eta\left(\frac{1+\sqrt{-15}}{4}\right)\right|=(30 \pi)^{-1 / 4} E^{1 / 8}\left(\frac{1+\sqrt{5}}{2}\right)^{1 / 12} .
$$

Hence we have proved the following result.

\section{Corollary 9.5}

$$
\begin{aligned}
& \eta\left(\frac{1+\sqrt{-15}}{2}\right)=\mathrm{e}^{\pi \mathrm{i} / 24}\left|\eta\left(\frac{1+\sqrt{-15}}{2}\right)\right| \\
&=\mathrm{e}^{\pi \mathrm{i} / 24}(30 \pi)^{-1 / 4} \mathrm{E}^{1 / 8}\left(\frac{1+\sqrt{5}}{2}\right)^{-1 / 12}, \\
&\left|\eta\left(\frac{1+\sqrt{-15}}{4}\right)\right|=(15 \pi)^{-1 / 4} \mathrm{E}^{1 / 8}\left(\frac{1+\sqrt{5}}{2}\right)^{1 / 12}
\end{aligned}
$$

Now we treat $d=-31$.

Example 9.6 $d=-31$. In this case we have

$$
H(-31)=\left\{1, A, A^{2}\right\}, \quad A^{3}=I,
$$

where

$$
\mathrm{I}=[1,1,8], \quad \mathrm{A}=[2,1,4], \quad \mathrm{A}^{2}=[2,-1,4]
$$


so that $h(-31)=3$. Also

$$
\begin{aligned}
\mathrm{K}_{31} & =[31,31,8]=[8,-31,31]=[8,1,1] \\
& =[1,-1,8]=[1,1,8]=\mathrm{I} .
\end{aligned}
$$

Next, with $A_{1}=A, h_{1}=3, \ell=1$, we have from (2.20)

$$
\begin{gathered}
{[I, I]=[I, A]=\left[I, A^{2}\right]=[A, I]=\left[A^{2}, I\right]=0,} \\
{[A, A]=1 / 3, \quad\left[A, A^{2}\right]=\left[A^{2}, A\right]=2 / 3, \quad\left[A^{2}, A^{2}\right]=4 / 3,}
\end{gathered}
$$

so that by $(2.21)$

$$
\begin{gathered}
f(I, I)=f(I, A)=f\left(I, A^{2}\right)=f(A, I)=f\left(A^{2}, I\right)=1, \\
f(A, A)=f\left(A^{2}, A^{2}\right)=\omega, \quad f\left(A, A^{2}\right)=f\left(A^{2}, A\right)=\omega^{2},
\end{gathered}
$$

where $\omega=e^{2 \pi i / 3}=\frac{1}{2}(-1+i \sqrt{3})$. From (2.33), we obtain for $r=0,1,2$

$$
\begin{aligned}
\ell\left(A^{r},-31\right) & =\prod_{\substack{p \\
\left(\frac{-31}{p}\right)=0}}\left(1+\frac{f\left(A^{r}, K_{p}\right)}{p}\right)=1+\frac{f\left(A^{r}, K_{31}\right)}{31}=1+\frac{f\left(A^{r}, I\right)}{31} \\
& =1+\frac{1}{31}=\frac{32}{31} .
\end{aligned}
$$

It is convenient to set

$$
\lambda(p):= \begin{cases}1, & \text { if }\left(\frac{-31}{p}\right)=1, K_{p}=1 \\ \omega, & \text { if }\left(\frac{-31}{p}\right)=1, K_{p}=A \\ \omega^{2}, & \text { if }\left(\frac{-31}{p}\right)=1, K_{p}=A^{2} \\ 0, & \text { otherwise. }\end{cases}
$$

Then, from (6.4), for $r=1,2$ we have

$$
\begin{aligned}
m\left(A^{r},-31\right) & =\lim _{s \rightarrow 1^{+}} \prod_{\substack{p \\
\left(\frac{-31}{p}\right)=1}} \frac{\left(1-\frac{1}{p^{s}}\right)\left(1+\frac{1}{p^{s}}\right)}{\left.\left(1-\frac{f\left(A^{r}, K_{p}\right)}{p^{s}}\right)\left(1-\frac{f\left(A^{r}, K_{p}\right)}{p}\right)^{-1}\right)} \\
& =\lim _{s \rightarrow 1^{+}} \prod_{\substack{p \\
\left(\frac{-31}{p}\right)=1}} \frac{\left(1-\frac{1}{p^{s}}\right)\left(1+\frac{1}{p^{s}}\right)}{\left(1-\frac{\lambda(p)}{p^{s}}\right)\left(1-\frac{\lambda(p)^{2}}{p^{s}}\right)}
\end{aligned}
$$

Appealing to Theorem 9.3 with $(a, b, c)=(1,1,8),(2,1,4)$ and $(2,-1,4)$, we obtain

$$
\left|\eta\left(\frac{1+\sqrt{-31}}{2}\right)\right|=(62 \pi)^{-1 / 4} \mathrm{Ee}^{-2 R}
$$


and

$$
\left|\eta\left(\frac{1+\sqrt{-31}}{4}\right)\right|=\left|\eta\left(\frac{-1+\sqrt{-31}}{4}\right)\right|=(31 \pi)^{-1 / 4} \mathrm{Ee}^{\mathrm{R}},
$$

where

$$
E:=\left\{\prod_{m=1}^{31} \Gamma\left(\frac{m}{31}\right)^{\left(\frac{-31}{m}\right)}\right\}^{1 / 12}
$$

and

$$
R:=\frac{4 \pi \sqrt{31}}{279} \lim _{s \rightarrow 1^{+}} \prod_{\substack{p \\\left(\frac{-31}{p}\right)=1}} \frac{\left(1-\frac{1}{p^{s}}\right)\left(1+\frac{1}{p^{s}}\right)}{\left(1-\frac{\lambda(p)}{p^{s}}\right)\left(1-\frac{\lambda(p)^{2}}{p^{s}}\right)}
$$

Hence we have the following result.

\section{Corollary 9.7}

$$
\eta\left(\frac{1+\sqrt{-31}}{2}\right)=\mathrm{e}^{\pi \mathrm{i} / 24}\left|\eta\left(\frac{1+\sqrt{-31}}{2}\right)\right|=\mathrm{e}^{\pi \mathrm{i} / 24}(62 \pi)^{-1 / 4} \mathrm{Ee}^{-2 \mathrm{R}}
$$

and

$$
\left|\eta\left(\frac{1+\sqrt{-31}}{4}\right)\right|=\left|\eta\left(\frac{-1+\sqrt{-31}}{4}\right)\right|=(31 \pi)^{-1 / 4} \mathrm{Ee}^{\mathrm{R}},
$$

where $E$ is defined in (9.2) and $R$ is defined in (9.3).

\section{Chowla-Selberg Formula}

In this section we recover the Chowla-Selberg formula [8] from Theorem 9.3.

Theorem 10.1 (Chowla-Selberg formula)

$$
\prod_{[a, b, c] \in H(d)} a^{-1 / 4}\left|\eta\left(\frac{b+\sqrt{d}}{2 a}\right)\right|=(2 \pi|d|)^{-h(d) / 4}\left\{\prod_{m=1}^{|d|} \Gamma\left(\frac{m}{|d|}\right)^{\left(\frac{d}{m}\right)}\right\}^{w(d) / 8} .
$$

Proof Multiplying formula (9.1) together over the $h(d)$ classes $K=[a, b, c]$ of $H(d)$, we obtain

$$
\begin{aligned}
& \prod_{[a, b, c] \in H(d)} a^{-1 / 4}\left|\eta\left(\frac{b+\sqrt{d}}{2 a}\right)\right|=(2 \pi|d|)^{-h(d) / 4}\left\{\prod_{m=1}^{|d|} \Gamma\left(\frac{m}{|d|}\right)^{\left(\frac{d}{m}\right)}\right\}^{w(d) / 8} \\
& e^{-\frac{\pi W(d)}{48 h(d)}} \sum_{K \in H(d)} \sum_{\substack{L \in H(d) \\
L \neq 1}} f(L, K) \ell(L, d) m(L, d)
\end{aligned}
$$


The result now follows as

$$
\begin{aligned}
\sum_{K \in H(d)} \sum_{\substack{L \in H(d) \\
L \neq 1}} f(L, K) \ell(L, d) m(L, d) & =\sum_{\substack{L \in H(d) \\
L \neq 1}} \ell(L, d) m(L, d) \sum_{K \in H(d)} f(L, K) \\
& =0,
\end{aligned}
$$

by (2.22).

\section{Chowla-Selberg Formula for G enera}

In this section we deduce from Theorem 9.3 the Chowla-Selberg formula for genera in the case of a fundamental discriminant $d$. This formula was first discovered by Williams and Zhang [10] in 1993, see also [5, formula (1.7)]. Note that in this formula $\Delta$ should be replaced by $d$ in 5 places and the subscript 1 in $w\left(d_{1}\right)$ should be deleted. For the basic properties of genera and generic characters, the reader is referred to [5, Section 2]. We will use the notation and terminology of [5] throughout this section.

Let $G$ be a genus of classes of $H(d)$, that is, $G$ is a coset of the subgroup $H^{2}(d)$ of squares in $H(d)$. The group of genera of discriminant $d$ is denoted by $G(d)$. The order of $G(d)$ is $2^{t}$, wheret $=t(d)$ is a nonnegative integer such that $t+1$ is the number of distinct prime divisors of $d$. Here we have made use of the fact that $d$ is a fundamental discriminant. Each genus contains $\frac{|H(d)|}{|G(d)|}=\frac{h(d)}{2^{t}}$ classes, so that

$$
|G|=h(d) / 2^{t} .
$$

By Theorem 9.3 and (11.1), we have

$$
\begin{aligned}
& \prod_{K=[a, b, c] \in G} a^{-1 / 4}\left|\eta\left(\frac{b+\sqrt{d}}{2 a}\right)\right|=\prod_{K \in G}\left\{2 \pi|d|^{-1 / 4}\left\{\prod_{m=1}^{|d|} \Gamma\left(\frac{m}{|d|}\right)^{\left(\frac{d}{m}\right)}\right\}^{w(d) / 8 h(d)}\right.
\end{aligned}
$$

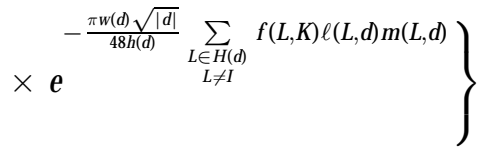

$$
\begin{aligned}
& =(2 \pi|d|)^{-h(d) / 2^{t+2}}\left\{\prod_{m=1}^{|d|} \Gamma\left(\frac{m}{|d|}\right)^{\left(\frac{d}{m}\right)}\right\}^{\frac{w(d)}{2+3}} \\
& \times e^{-\frac{\pi w(d) \sqrt{|d|}}{48 h(d)}} \sum_{K \in G} \sum_{L \neq 1} f(L, K) \ell(L, d) m(L, d),
\end{aligned}
$$

so that

$$
\begin{aligned}
& \prod_{[a, b, c] \in G} a^{-1 / 4}\left|\eta\left(\frac{b+\sqrt{d}}{2 a}\right)\right| \\
& =(2 \pi|d|)^{-h(d) / 2^{t+2}}\left\{\prod_{m=1}^{|d|} \Gamma\left(\frac{m}{|d|}\right)^{\left(\frac{d}{m}\right)}\right\}^{\frac{w(d)}{2 t+3}} e^{-\frac{\pi w(d) \sqrt{(d \mid}}{48 h(d)} E}
\end{aligned}
$$


where

$$
E=\sum_{L \neq 1} \ell(L, d) m(L, d) \sum_{K \in G} f(L, K) .
$$

In order to determine $\sum_{K \in G} f(L, K)$ we recall from (2.17) and (2.18) that $\left\{A_{1}, \ldots, A_{\ell}\right\}$ is a set of generators of $H(d)$ such that the $h_{i}=\operatorname{ord~} A_{i}(i=1, \ldots, \ell)$ satisfy $h_{1}\left|h_{2}\right| \cdots \mid h_{\ell}$. Thus we may suppose that $h_{1}, \ldots, h_{m}$ are odd and $h_{m+1}, \ldots, h_{\ell}$ are even, where $m$ is an integer satisfying $0 \leq \mathrm{m} \leq \ell$. The genera of $\mathrm{H}(\mathrm{d})$ are given by

$$
A_{m+1}^{r_{m+1}} \cdots A_{\ell}^{r_{\ell}} H^{2}(d)
$$

with $r_{m+1}, \ldots, r_{\ell}=0$ or 1 . There are $2^{\ell-m}$ genera in total so that

$$
\mathrm{t}=\ell-\mathrm{m}
$$

We suppose that the genus $G$ is given by

$$
\mathrm{G}=\mathrm{A}_{\mathrm{m}+1}^{\varepsilon_{\mathrm{m}+1}} \cdots \mathrm{A}_{\ell}^{\varepsilon_{\ell}} \mathrm{H}^{2}(\mathrm{~d}), \quad \text { where each } \varepsilon_{\mathrm{m}+1}, \ldots, \varepsilon_{\ell}=0 \text { or } 1 \text {. }
$$

The classes $\mathrm{K}$ of the genus $\mathrm{G}$ are given by

$$
K=A_{1}^{k_{1}} \cdots A_{m}^{k_{m}} A_{m+1}^{2 k_{m+1}+\varepsilon_{m+1}} \cdots A_{\ell}^{2 k_{\ell}+\varepsilon_{\ell}}
$$

where

$$
k_{j}=\left\{\begin{array}{l}
0,1, \ldots, h_{j}-1 ; j=1, \ldots, m, \\
0,1, \ldots,\left(h_{j} / 2\right)-1 ; j=m+1, \ldots, \ell .
\end{array}\right.
$$

Thus, for $L \in H(d)$, say $L=A_{1}^{t_{1}} \cdots A_{\ell}^{t_{\ell}}, 0 \leq t_{j}<h_{j}, j=1, \ldots, \ell$, we have

$$
[L, K]=\sum_{j=1}^{m} \frac{t_{j} k_{j}}{h_{j}}+\sum_{j=m+1}^{\ell} \frac{t_{j}\left(2 k_{j}+\varepsilon_{j}\right)}{h_{j}},
$$

so that appealing to (2.17), (2.21), (11.4), (11.6), (11.7) and (11.8), we obtain

$$
\begin{aligned}
\sum_{K \in G} f(L, K) & \\
= & \sum_{K \in G} e^{2 \pi i[L, K]} \\
& =\prod_{j=1}^{m}\left\{\sum_{k_{j}=0}^{h_{j}-1} e^{\frac{2 \pi i i_{j} k_{j}}{h_{j}}}\right\} \prod_{j=m+1}^{\ell}\left\{\sum_{k_{j}=0}^{\left(h_{j} / 2\right)-1} e^{\frac{2 \pi i_{j}\left(2 k_{j}+\varepsilon_{j}\right)}{h_{j}}}\right\} \\
= & \prod_{j=1}^{m}\left\{\begin{array}{cc}
h_{j}, & \text { if } t_{j}=0 \\
0, & \text { if } t_{j} \neq 0
\end{array}\right\} \prod_{j=m+1}^{\ell} e^{\frac{2 \pi i i_{j} \varepsilon_{j}}{h_{j}}} \prod_{j=m+1}^{\ell}\left\{\sum_{k_{j}=0}^{\left(h_{j} / 2\right)-1} e^{\frac{2 \pi i t_{j} k_{j}}{\left(h_{j} / 2\right)}}\right\}
\end{aligned}
$$




$$
\begin{aligned}
& =e^{2 \pi i} \sum_{j=m+1}^{\ell} \prod_{j=1}^{t_{j} \varepsilon_{j}} \prod_{j}^{m}\left\{\begin{array}{cc}
h_{j}, & \text { if } t_{j}=0 \\
0, & \text { if } t_{j} \neq 0
\end{array}\right\} \prod_{j=m+1}^{\ell}\left\{\begin{array}{cc}
h_{j} / 2, & \text { if } t_{j}=0 \text { or } h_{j} / 2 \\
0, & \text { if } t_{j} \neq 0, h_{j} / 2
\end{array}\right\} \\
& = \begin{cases}e^{2 \pi i} \sum_{j=m+1}^{\ell} \frac{t_{j} \varepsilon_{j}}{h_{j}} h_{1} \cdots h_{m} / 2^{\ell-m}, & \text { if } t_{j}=0(1 \leq j \leq m) \\
0, & \text { and } t_{j}=\frac{h_{j} x_{j}}{2}(m+1 \leq j \leq \ell) \\
0, & \text { and each } x_{j}=0 \text { or } 1,\end{cases}
\end{aligned}
$$

that is

$$
\begin{aligned}
& \sum_{K \in G} f(L, K) \\
& \quad= \begin{cases}\frac{h(d)}{2^{t}}(-1)^{\sum_{j=m+1}^{\ell} x_{j} \varepsilon_{j}}, & \text { if } L=\prod_{j=m+1}^{\ell} A_{j}^{h_{j} x_{j} / 2}, \text { where each } x_{j}=0 \text { or } 1, \\
0, & \text { otherwise. }\end{cases}
\end{aligned}
$$

Hence, from (11.3) and (11.9), we obtain

$$
E=\frac{h(d)}{2^{t}} \sum_{x_{m+1}, \ldots, x_{\ell}=0}^{1}(-1)^{\sum_{j=m+1}^{\ell} x_{j} \varepsilon_{j}} \ell\left(\prod_{j=m+1}^{\ell} A_{j}^{h_{j} x_{j} / 2}, d\right) m\left(\prod_{j=m+1}^{\ell} A_{j}^{h_{j} x_{j} / 2}, d\right),
$$

where the prime $(')$ indicates that $\left(x_{m+1}, \ldots, x_{\ell}\right)=(0, \ldots, 0)$ is omitted.

Next we turn to the evaluation of the terms appearing in the sum on the right side of (11.10). We denotethe distinct primes dividing $d$ by $p_{1}, \ldots, p_{t+1}$ and the corresponding prime discriminants by $p_{1}^{*}, \ldots, p_{t+1}^{*}$, see $D$ efinition 2.1 of $[5]$, so that

$$
d=p_{1}^{*} \cdots p_{t+1}^{*} .
$$

We define the sets $F(d), F_{+}(d)$ and $F_{-}(d)$ as follows:

$$
\begin{gathered}
F(d):=\left\{d_{1} \mid d_{1}=p_{1}^{* y_{1}} \cdots p_{t+1}^{* y_{t+1}}, y_{1}, \ldots, y_{t+1}=0 \text { or } 1\right\}, \\
F_{+}(d):=\left\{d_{1} \in F(d) \mid d_{1}>0\right\} \\
F_{-}(d):=\left\{d_{1} \in F(d) \mid d_{1}<0\right\} .
\end{gathered}
$$

Clearly, from (11.12), (11.13) and (11.14), we see that

$$
F(d)=F_{+}(d) \cup F_{-}(d), \quad F_{+}(d) \cap F_{-}(d)=\varnothing, \quad|F(d)|=2^{t+1} .
$$

M oreover the mapping $\theta: F_{+}(d) \rightarrow F_{-}(d)$ given by $\theta\left(d_{1}\right)=d / d_{1}$ is a bijection so that

$$
\left|F_{+}(d)\right|=\left|F_{-}(d)\right| \text {. }
$$


Hence, from (11.15) and (11.16), we deduce that

$$
\left|F_{+}(d)\right|=\frac{1}{2}|F(d)|=2^{t} .
$$

Now set

$$
X:=\{0,1\}^{\ell-m}
$$

so that by (11.4) and (11.17)

$$
|X|=2^{\ell-m}=2^{t}=\left|F_{+}(d)\right|,
$$

and define a mapping

$$
\lambda: \mathrm{F}_{+}(\mathrm{d}) \rightarrow \mathrm{X}
$$

by

$$
\lambda\left(d_{1}\right)=\left(x_{m+1}, \ldots, x_{\ell}\right)
$$

where

$$
\gamma_{d_{1}}\left(A_{j}\right)=(-1)^{x_{j}}, \quad x_{j}=0 \text { or } 1, \quad j=m+1, \ldots, \ell .
$$

We show that $\lambda$ is a bijection. By (11.18) it suffices to show that $\lambda$ is one-to-one. Let $\mathrm{d}_{1}, \mathrm{~d}_{1}^{\prime} \in \mathrm{F}_{+}(\mathrm{d})$ be such that

$$
\lambda\left(d_{1}\right)=\lambda\left(d_{1}^{\prime}\right) .
$$

Then

$$
\gamma_{d_{1}}\left(A_{j}\right)=\gamma_{d_{1}^{\prime}}\left(A_{j}\right), \quad j=m+1, \ldots, \ell .
$$

Hence

$$
\gamma_{\Delta\left(d_{1} d_{1}^{\prime}\right)}\left(A_{j}\right)=\gamma_{d_{1} d_{1}^{\prime}}\left(A_{j}\right)=\gamma_{d_{1}}\left(A_{j}\right) \gamma_{d_{1}^{\prime}}\left(A_{j}\right)=1, \quad j=m+1, \ldots, \ell .
$$

This shows that

$$
\gamma_{\Delta\left(d_{1} d_{1}^{\prime}\right)}\left(G_{1}\right)=1, \quad \text { for all genera } G_{1} \text { of } H(d)
$$

Hence

$$
\Delta\left(\mathrm{d}_{1} \mathrm{~d}_{1}^{\prime}\right)=1 \text { or } \mathrm{d}
$$

that is

$$
\mathrm{d}_{1}^{\prime}=\mathrm{d}_{1} \text { or } \mathrm{d}_{1}^{\prime}=\mathrm{d} / \mathrm{d}_{1} \text {. }
$$

The latter possibility cannot occur as $d_{1}>0, d_{1}^{\prime}>0, d<0$. Hence $\lambda$ is a bijection. Thus the inverse map $\lambda^{-1}: X \rightarrow F_{+}(d)$ associates with each $\ell-m$ tuple $\left(x_{m+1}, \ldots, x_{\ell}\right)$ (each $\mathrm{x}_{\mathrm{j}}=0$ or 1) a unique positive fundamental discriminant $\mathrm{d}_{1}$ such that $\gamma_{\mathrm{d}_{1}}\left(\mathrm{~A}_{\mathrm{j}}\right)=(-1)^{\mathrm{x}_{j}}$ $(j=m+1, \ldots, \ell)$. Further $\lambda^{-1}((0, \ldots, 0))=1$.

$$
\begin{aligned}
& \text { Wenow consider }(-1)^{\sum_{j=m+1}^{\ell} x_{j} \varepsilon_{j}} \text {. We have } \\
& \qquad(-1)^{\sum_{j=m+1}^{\ell} x_{j} \varepsilon_{j}}=\prod_{j=m+1}^{\ell}\left((-1)^{x_{j}}\right)^{\varepsilon_{j}}
\end{aligned}
$$




$$
\begin{aligned}
& =\prod_{j=m+1}^{\ell} \gamma_{d_{1}}\left(A_{j}\right)^{\varepsilon_{j}} \\
& =\prod_{j=m+1}^{\ell} \gamma_{d_{1}}\left(A_{j}^{\varepsilon_{j}}\right) \\
& =\gamma_{d_{1}}\left(\prod_{j=m+1}^{\ell} A_{j}^{\varepsilon_{j}}\right),
\end{aligned}
$$

that is

$$
(-1)^{\sum_{j=m+1}^{\ell} x_{j} \varepsilon_{j}}=\gamma_{j_{1}}(G)
$$

Next we consider $\ell\left(\prod_{j=m+1}^{\ell} A_{j}^{h_{j} x_{j} / 2}, d\right)$. For $s=1, \ldots, t+1$ we have $p_{s} \mid d$ so that $K_{p_{s}}=$ $\mathrm{K}_{\mathrm{p}_{\mathrm{s}}}^{-1}$ and thus

$$
K_{p_{s}}=\prod_{j=m+1}^{\ell} A_{j}^{h_{j} u_{j s} / 2}
$$

where

$$
u_{j s}=0 \text { or } 1 ; \quad j=m+1, \ldots, \ell, \quad s=1, \ldots, t+1
$$

Hence

$$
\begin{aligned}
f\left(\prod_{j=m+1}^{\ell} A_{j}^{h_{j} x_{j} / 2}, K_{p_{s}}\right) & =f\left(\prod_{j=m+1}^{\ell} A_{j}^{h_{j} x_{j} / 2}, \prod_{j=m+1}^{\ell} A_{j}^{h_{j} u_{j} / 2}\right) \\
& =e^{2 \pi i\left[\prod_{j=m+1}^{\ell} A_{j}^{h_{j} x_{j} / 2}, \prod_{j=m+1}^{\ell} A_{j}^{h_{j} u_{j} / 2}\right]} \\
& =e^{2 \pi i \sum_{j=m+1}^{\ell} \frac{\left(h_{j} x_{j} / 2\right)\left(h_{j} u_{j s} / 2\right)}{h_{j}}} \\
& =(-1)^{j=m+1} \sum_{j}^{\ell} h_{j} x_{j} u_{j} / 2 \\
& =\prod_{j=m+1}^{\ell}\left((-1)^{x_{j}}\right)^{h_{j} u_{j s} / 2} \\
& =\prod_{j=m+1}^{\ell}\left(\gamma_{d_{1}}\left(A_{j}\right)\right)^{h_{j} u_{j s} / 2} \\
& =\gamma_{d_{1}}\left(\prod_{j=m+1}^{\ell} A_{j}^{h_{j} u_{j} / 2}\right)
\end{aligned}
$$




$$
=\gamma_{d_{1}}\left(K_{p_{s}}\right)
$$

If $p_{s} \nmid d_{1}$ then $\gamma_{d_{1}}\left(K_{p_{s}}\right)=\left(\frac{d_{1}}{p_{s}}\right)$. If $p_{s} \mid d_{1}$ then $p_{s} \nmid d / d_{1}$ and $\gamma_{d_{1}}\left(K_{p_{s}}\right)=\gamma_{d / d_{1}}\left(K_{p_{s}}\right)=\left(\frac{d / d_{1}}{p_{s}}\right)$. Hence

$$
\begin{aligned}
\ell\left(\prod_{j=m+1}^{\ell} A_{h_{j}}^{h_{j} x_{j} / 2}, d\right) & =\prod_{\substack{p \\
\left(\frac{d}{p}\right)=0}}\left(1+\frac{f\left(\prod_{j=m+1}^{\ell} A_{j}^{h_{j} x_{j} / 2}, K_{p}\right)}{p}\right) \\
& =\prod_{s=1}^{t+1}\left(1+\frac{f\left(\prod_{j=m+1}^{\ell} A_{j}^{h_{j} x_{j} / 2}, K_{p_{s}}\right)}{p_{s}}\right) \\
& =\prod_{s=1}^{t+1}\left(1+\frac{\left(\frac{d_{1}}{p_{s}}\right)}{p_{s}}\right) \prod_{\substack{s=1 \\
p_{s} \nmid d_{1}}}^{t+1}\left(1+\frac{\left(\frac{d / d_{1}}{p_{s}}\right)}{p_{s}}\right) \\
& =\prod_{p \mid d}\left(1+\frac{\left(\frac{d_{1}}{p}\right)}{p}\right)\left(1+\frac{\left(\frac{d / d_{1}}{p}\right)}{p}\right) \\
& =\prod_{p \mid d} \frac{\left(1-\frac{1}{p}\right)\left(1+\frac{1}{p}\right)}{\left(1-\frac{\left(\frac{d_{1}}{p}\right)}{p}\right)\left(1-\frac{\left(\frac{d / d_{1}}{p}\right)}{p}\right)},
\end{aligned}
$$

that is

$$
\ell\left(\prod_{j=m+1}^{\ell} A_{j}^{h_{j} x_{j} / 2}, d\right)=\lim _{s \rightarrow 1^{+}} \prod_{\substack{p \\\left(\frac{d}{p}\right)=0}} \frac{\left(1-\frac{1}{p^{s}}\right)\left(1+\frac{1}{p^{s}}\right)}{\left(1-\frac{\left(\frac{d}{p}\right)}{p^{s}}\right)\left(1-\frac{\left(\frac{d / d_{1}}{p}\right)}{p^{s}}\right)} .
$$

Now we consider $m\left(\prod_{j=m+1}^{\ell} A_{j}^{h_{j} x_{j} / 2}, d\right)$. Let $p$ be a prime such that $\left(\frac{d}{p}\right)=1$ so that $K_{p} \in H(d)$. Set

$$
K_{p}=\prod_{j=1}^{\ell} A_{j}^{z_{j}}, \quad z_{j}=0,1, \ldots, h_{j}-1, j=1, \ldots, \ell
$$

Then

$$
\begin{aligned}
f\left(\prod_{j=m+1}^{\ell} A_{j}^{\frac{h_{j} x_{j}}{2}}, K_{p}\right) & =f\left(\prod_{j=m+1}^{\ell} A_{j}^{h_{j} x_{j} / 2}, \prod_{j=1}^{\ell} A_{j}^{z_{j}}\right) \\
& =e^{2 \pi i\left[\prod_{j=m+1}^{\ell} A_{j}^{h_{j} x_{j} / 2}, \prod_{j=1}^{\ell} A_{j}^{z_{j}}\right]}
\end{aligned}
$$




$$
\begin{aligned}
& =e^{2 \pi i} \sum_{j=m+1}^{\ell} \frac{\left(h_{j} x_{j} / 2\right)\left(z_{j}\right)}{h_{j}} \\
& =\prod_{j=m+1}^{\ell}\left((-1)^{\sum_{j=m+1}^{\ell} x_{j} z_{j}}\right)^{z_{j}} \\
& =\prod_{j=m+1}^{\ell}\left(\gamma_{d_{1}}\left(A_{j}\right)\right)^{z_{j}} \\
& =\prod_{j=m+1}^{\ell} \gamma_{d_{1}}\left(A_{j}^{z_{j}}\right) \\
& =\gamma_{d_{1}}\left(\prod_{j=m+1}^{\ell} A_{j}^{z_{j}}\right) \\
& =\gamma_{d_{1}}\left(K_{p}\right) \\
& =\left(\frac{d_{1}}{p}\right)=\left(\frac{d / d_{1}}{p}\right) .
\end{aligned}
$$

Thus by (6.4) we obtain

$$
m\left(\prod_{j=m+1}^{\ell} A_{j}^{h_{j} x_{j} / 2}, d\right)=\lim _{s \rightarrow 1^{+}} \prod_{\substack{p \\\left(\frac{d}{p}\right)=1}} \frac{\left(1-\frac{1}{p^{s}}\right)\left(1+\frac{1}{p^{s}}\right)}{\left(1-\frac{\left(\frac{d_{1}}{p}\right)}{p^{s}}\right)\left(1-\frac{\left(\frac{d / d_{1}}{p}\right)}{p^{s}}\right)} .
$$

Since

$$
\prod_{\substack{p \\\left(\frac{d}{p}\right)=-1}} \frac{\left(1-\frac{1}{p^{s}}\right)\left(1+\frac{1}{p^{s}}\right)}{\left(1-\frac{\left(\frac{d_{1}}{p}\right)}{p^{s}}\right)\left(1-\frac{\left(\frac{d / d_{1}}{p}\right)}{p^{s}}\right)}=1,
$$

we deduce from (11.23), (11.24) and (11.25) that

$$
\begin{aligned}
\ell\left(\prod_{j=m+1}^{\ell} A_{j}^{h_{j} x_{j} / 2}, d\right) m\left(\prod_{j=m+1}^{\ell} A_{j}^{h_{j} x_{j} / 2}, d\right) & =\lim _{s \rightarrow 1^{+}} \prod_{p} \frac{\left(1-\frac{1}{p^{s}}\right)\left(1+\frac{1}{p^{s}}\right)}{\left(1-\frac{\left(\frac{d_{1}}{p}\right)}{p^{s}}\right)\left(1-\frac{\left(\frac{d / d_{1}}{p}\right)}{p^{s}}\right)} \\
& =\lim _{s \rightarrow 1^{+}} \frac{L\left(s, d_{1}\right), L\left(s, d / d_{1}\right)}{\zeta(2 s)},
\end{aligned}
$$

that is

(11.26)

$$
\ell\left(\prod_{j=m+1}^{\ell} A_{j}^{h_{j} x_{j} / 2}, d\right) m\left(\prod_{j=m+1}^{\ell} A_{j}^{h_{j} x_{j} / 2}, d\right)=\frac{6}{\pi^{2}} L\left(1, d_{1}\right) L\left(1, d / d_{1}\right) .
$$


Hence, by (11.22) and (11.26), we obtain

$$
\begin{aligned}
& \sum_{x_{m+1}, \ldots, x_{\ell}=0}^{1}(-1)^{\sum_{j=m+1}^{\ell} x_{j} \varepsilon_{j}} \ell\left(\prod_{j=m+1}^{\ell} A_{j}^{h_{j} x_{j} / 2}, d\right) m\left(\prod_{j=m+1}^{\ell} A_{j}^{h_{j} x_{j} / 2}, d\right) \\
& =\frac{6}{\pi^{2}} \sum_{\substack{d_{1} \in F_{+}(d) \\
d_{1} \neq 1}} \gamma_{d_{1}}(G) L\left(1, d_{1}\right) L\left(1, d / d_{1}\right) \\
& =\frac{6}{\pi^{2}} \sum_{1<d_{1} \in F(d)} \gamma_{d_{1}}(G) \frac{2 h\left(d_{1}\right) \log \varepsilon_{d_{1}}}{\sqrt{d_{1}}} \frac{2 \pi h\left(d / d_{1}\right)}{W\left(d / d_{1}\right) \sqrt{\left|d / d_{1}\right|}} \\
& =\frac{24}{\pi \sqrt{|d|}} \sum_{1<d_{1} \in F(d)} \gamma_{d_{1}}(G) \frac{h\left(d_{1}\right) h\left(d / d_{1}\right)}{w\left(d / d_{1}\right)} \log \varepsilon_{d_{1}},
\end{aligned}
$$

so that by (11.10)

$$
E=\frac{24 h(d)}{2^{t} \pi \sqrt{|d|}} \sum_{1<d_{1} \in F(d)} \gamma_{d_{1}}(G) \frac{h\left(d_{1}\right) h\left(d / d_{1}\right)}{w\left(d / d_{1}\right)} \log \varepsilon_{d_{1}} .
$$

Finally

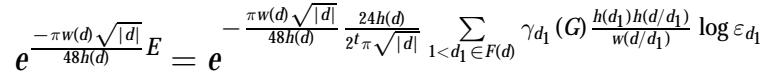

$$
\begin{aligned}
& =e^{-\sum_{1<d_{1} \in F(d)} \frac{w(d) / d_{1}(G) h\left(d_{1}\right) h\left(d / d_{1}\right)}{w\left(d / d_{1}\right) 2^{l+1}} \log \varepsilon_{d_{1}}} \\
& =\prod_{1<d_{1} \in F(d)} \varepsilon_{d_{1}}^{-\frac{w(d) / \gamma_{1}(G) h\left(d_{1}\right) h\left(d / d_{1}\right)}{w\left(d / d d_{1}\right) 2^{2+1}}} .
\end{aligned}
$$

This completes the proof of the Chowla-Selberg formula for genera as given by Williams and Zhang in the case of a fundamental discriminant.

Theorem 11.1 Let $\mathrm{G}$ be a genus of classes of $\mathrm{H}(\mathrm{d})$. Then

$$
\begin{gathered}
\prod_{[a, b, c] \in G} a^{-1 / 4}\left|\eta\left(\frac{b+\sqrt{d}}{2 a}\right)\right|=(2 \pi|d|)^{-h(d) / 2^{t+2}}\left\{\prod_{m=1}^{|d|} \Gamma\left(\frac{m}{|d|}\right)^{\left(\frac{d}{m}\right)}\right\}^{\frac{w(d)}{2^{t+3}}} \\
\times \prod_{\substack{d_{1} \in F(d) \\
d_{1}>1}} \varepsilon_{d_{1}}^{-\frac{w(d) / d_{1}(G) h\left(d_{1}\right) h\left(d / d_{1}\right)}{w\left(d / d_{1}\right) 2^{t+1}}},
\end{gathered}
$$

where $\varepsilon_{d_{1}}$ denotes the fundamental unit ( $>1$ ) of the real quadratic field $Q\left(\sqrt{d_{1}}\right), \gamma_{d_{1}}(G)(=$ \pm 1 ) is defined in [5, Section 2], $F(d)$ is defined in (11.12), and $t+1$ is the number of distinct prime factors of the fundamental discriminant $d$. 


\section{Concluding remarks}

Let $K \in H(d)$. It is clear from Theorem 9.3 that the sum

$$
\sum_{\substack{L \in H(d) \\ L \neq 1}} f(L, K) \ell(L, d) m(L, d)
$$

does not depend upon the choice of basis $\mathcal{A}=\left\{\mathrm{A}_{1}, \ldots, \mathrm{A}_{\ell}\right\}$ of $\mathrm{H}(\mathrm{d})$. In this section we prove this assertion directly (Theorem 12.4). Let $\mathcal{B}=\left\{B_{1}, \ldots, B_{\ell}\right\}$ be another basis of $H(d)$ with $\operatorname{ord}\left(B_{i}\right)=h_{i}(i=1, \ldots, \ell)$. Hence there exist unique integers $a_{i j}(i, j=$ $1, \ldots, \ell)$ such that

$$
B_{i}=\prod_{j=1}^{\ell} A_{j}^{a_{i j}}, \quad 0 \leq a_{i j}<h_{j},
$$

and unique integers $b_{i j}(i, j=1, \ldots, \ell)$ such that

$$
A_{i}=\prod_{j=1}^{\ell} B_{j}^{b_{i j}}, \quad 0 \leq b_{i j}<h_{j} .
$$

Now, by (12.2), we have

$$
\prod_{j=1}^{\ell} B_{j}^{b_{i j} h_{i}}=\left(\prod_{j=1}^{\ell} B_{j}^{b_{i j}}\right)^{h_{i}}=A_{i}^{h_{i}}=I,
$$

so that

$$
b_{i j} h_{i} \equiv 0 \quad\left(\bmod h_{j}\right), \quad i, j=1, \ldots, \ell
$$

Also, by (12.1), we have

$$
\prod_{j=1}^{\ell} A_{j}^{a_{i j} h_{i}}=\left(\prod_{j=1}^{\ell} A_{j}^{a_{i j}}\right)^{h_{i}}=B_{i}^{h_{i}}=I,
$$

so that

$$
a_{i j} h_{i} \equiv 0 \quad\left(\bmod h_{j}\right), \quad i, j=1, \ldots, \ell
$$

Further

$$
\prod_{k=1}^{\ell} A_{k}^{\sum_{j=1}^{\ell} a_{j k} b_{i j}}=\prod_{k=1}^{\ell} \prod_{j=1}^{\ell} A_{k}^{a_{j k} b_{i j}}=\prod_{j=1}^{\ell}\left(\prod_{k=1}^{\ell} A_{k}^{a_{j k}}\right)^{b_{i j}}=\prod_{j=1}^{\ell} B_{j}^{b_{i j}}=A_{i}
$$


so that

$$
\sum_{j=1}^{\ell} a_{j k} b_{i j} \equiv \delta_{i k} \quad\left(\bmod h_{k}\right), \quad i, k=1, \ldots, \ell
$$

Similarly we have

$$
\prod_{k=1}^{\ell} B_{k}^{\ell=1} a_{i j} b_{j k}=\prod_{k=1}^{\ell} \prod_{j=1}^{\ell} B_{k}^{a_{i j} b_{j k}}=\prod_{j=1}^{\ell}\left(\prod_{k=1}^{\ell} B_{k}^{b_{j k}}\right)^{a_{i j}}=\prod_{j=1}^{\ell} A_{j}^{a_{i j}}=B_{i},
$$

so that

$$
\sum_{j=1}^{\ell} a_{i j} b_{j k} \equiv \delta_{i k} \quad\left(\bmod h_{k}\right), \quad i, k=1, \ldots, \ell
$$

As $\mathrm{K} \in \mathrm{H}(\mathrm{d})$ we can define integers $\mathrm{k}_{\mathrm{i}}(\mathrm{i}=1, \ldots, \ell)$ and $\mathrm{k}_{\mathrm{i}}^{\prime}(\mathrm{i}=1, \ldots, \ell)$ by

$$
\mathrm{K}=\prod_{\mathrm{i}=1}^{\ell} \mathrm{A}_{\mathrm{i}}^{\mathrm{K}_{\mathrm{i}}}=\prod_{\mathrm{i}=1}^{\ell} \mathrm{B}_{\mathrm{i}}^{\mathrm{K}_{\mathrm{i}}^{\prime}}, \quad 0 \leq \mathrm{k}_{\mathrm{i}}, \mathrm{k}_{\mathrm{i}}^{\prime}<\mathrm{h}_{\mathrm{i}} .
$$

From (12.2) and (12.7), we obtain

$$
\prod_{j=1}^{\ell} B_{j}^{k_{j}^{\prime}}=\prod_{i=1}^{\ell}\left(\prod_{j=1}^{\ell} B_{j}^{b_{i j}}\right)^{k_{i}}=\prod_{j=1}^{\ell} B_{j}^{\ell=1} k_{i} b_{i j}
$$

so that

$$
k_{j}^{\prime} \equiv \sum_{i=1}^{\ell} k_{i} b_{i j} \quad\left(\bmod h_{j}\right), \quad j=1, \ldots, \ell .
$$

From (12.1) and (12.7) we have

$$
\prod_{j=1}^{\ell} A_{j}^{k_{j}}=\prod_{i=1}^{\ell}\left(\prod_{j=1}^{\ell} A_{j}^{a_{i j}}\right)^{k_{i}^{\prime}}=\prod_{j=1}^{\ell} A_{j}^{\ell=1} k_{i}^{\prime} a_{i j}
$$

so that

$$
k_{j} \equiv \sum_{i=1}^{\ell} k_{i}^{\prime} a_{i j} \quad\left(\bmod h_{j}\right)
$$


Now let $\mathrm{L} \in \mathrm{H}(\mathrm{d})$ and define integers $\ell_{\mathrm{i}}$ and $\ell_{\mathrm{i}}^{\prime}(\mathrm{i}=1, \ldots, \ell)$ by

$$
\mathrm{L}=\prod_{i=1}^{\ell} \mathrm{A}_{\mathrm{i}}^{\ell_{i}}=\prod_{i=1}^{\ell} \mathrm{B}_{\mathrm{i}}^{\ell_{i}^{\prime}}, \quad 0 \leq \ell_{\mathrm{i}}, \ell_{\mathrm{i}}^{\prime}<\mathrm{h}_{\mathrm{i}} .
$$

Set

$$
x_{i}=\sum_{j=1}^{\ell} b_{i j} \ell_{j}^{\prime} h_{i} / h_{j}, \quad i=1, \ldots, \ell .
$$

By $(12.3) x_{i}(i=1, \ldots, \ell)$ is a nonnegative integer. Set

$$
L^{*}=\prod_{i=1}^{\ell} A_{i}^{x_{i}} \in H(d) .
$$

Lemma $12.1\left[\mathrm{~L}^{*}, \mathrm{~K}\right]_{\mathcal{A}} \equiv[\mathrm{L}, \mathrm{K}]_{\mathcal{B}}(\bmod 1)$.

Proof Wehave

$$
\begin{aligned}
{\left[L^{*}, K\right]_{\mathcal{A}} } & =\sum_{i=1}^{\ell} \frac{x_{i} k_{i}}{h_{i}} \\
& =\sum_{i=1}^{\ell} \frac{k_{i}}{h_{i}} \sum_{j=1}^{\ell} b_{i j} \ell_{j}^{\prime} \frac{h_{i}}{h_{j}} \quad(\text { by (12.11)) } \\
& =\sum_{j=1}^{\ell} \frac{\ell_{j}^{\prime}}{h_{j}} \sum_{i=1}^{\ell} k_{i} b_{i j} \\
& \equiv \sum_{j=1}^{\ell} \frac{\ell_{j}^{\prime}}{h_{j}} k_{j}^{\prime} \quad(\bmod 1) \quad(\text { by }(12.8)) \\
& =[L, K]_{\mathcal{B}} .
\end{aligned}
$$

Lemma 12.2 Themapping $\theta: H(d) \rightarrow H(d)$ given by

$$
\theta(\mathrm{L})=\mathrm{L}^{*}
$$

is a bijection with $\theta(\mathrm{I})=\mathrm{I}$.

Proof Suppose $L_{1}, L_{2} \in H(d)$ are such that

$$
\theta\left(\mathrm{L}_{1}\right)=\theta\left(\mathrm{L}_{2}\right)
$$


For $r=1,2$ we set $\ell_{j}(r)=\operatorname{ind}_{A_{j}}\left(L_{r}\right)$ and $\ell_{j}^{\prime}(r)=\operatorname{ind}_{B_{j}}\left(L_{r}\right), j=1, \ldots, \ell$. Then, for $\mathrm{i}=1, \ldots, \ell$, we have

$$
\sum_{j=1}^{\ell} b_{i j} \ell_{j}^{\prime}(1) h_{i} / h_{j} \equiv \sum_{j=1}^{\ell} b_{i j} \ell_{j}^{\prime}(2) h_{i} / h_{j} \quad\left(\bmod h_{i}\right),
$$

so that

$$
\sum_{j=1}^{\ell} b_{i j}\left(\ell_{j}^{\prime}(1)-\ell_{j}^{\prime}(2)\right) / h_{j} \equiv 0 \quad(\bmod 1)
$$

Hence

$$
\sum_{i=1}^{\ell} a_{k i} \sum_{j=1}^{\ell} b_{i j}\left(\ell_{j}^{\prime}(1)-\ell_{j}^{\prime}(2)\right) / h_{j} \equiv 0 \quad(\bmod 1), \quad k=1, \ldots, \ell
$$

Thus

$$
\sum_{j=1}^{\ell} \frac{\left(\ell_{j}^{\prime}(1)-\ell_{j}^{\prime}(2)\right)}{h_{j}} \sum_{i=1}^{\ell} a_{k i} b_{i j} \equiv 0 \quad(\bmod 1), \quad k=1, \ldots, \ell .
$$

Appealing to (12.6) we obtain

$$
\sum_{j=1}^{\ell} \frac{\left(\ell_{j}^{\prime}(1)-\ell_{j}^{\prime}(2)\right)}{h_{j}} \delta_{j k} \equiv 0 \quad(\bmod 1)
$$

that is

$$
\frac{\ell_{\mathrm{k}}^{\prime}(1)-\ell_{\mathrm{k}}^{\prime}(2)}{h_{\mathrm{k}}} \equiv 0 \quad(\bmod 1), \quad \mathrm{k}=1, \ldots, \ell .
$$

Hence $\ell_{k}^{\prime}(1) \equiv \ell_{k}^{\prime}(2)\left(\bmod h_{k}\right)(k=1, \ldots, \ell)$ so that $L_{1}=L_{2}$. Thus $\theta$ is injective and so $\theta$ is a bijection. It is clear from (12.10), (12.11) and (12.12) that $\theta(\mathrm{I})=\mathrm{I}$.

\section{Lemma 12.3}

(i) $\mathrm{f}_{\mathcal{A}}\left(\mathrm{L}^{*}, \mathrm{~K}\right)=\mathrm{f}_{\mathcal{B}}(\mathrm{L}, \mathrm{K})$,

(ii) $\ell_{\mathcal{A}}\left(L^{*}, d\right)=\ell_{\mathcal{B}}(L, d)$,

(iii) $\mathrm{m}_{\mathcal{A}}\left(\mathrm{L}^{*}, \mathrm{~d}\right)=\mathrm{m}_{\mathcal{B}}(\mathrm{L}, \mathrm{d})$.

\section{Proof}

(i)

$$
\begin{array}{rlrl}
\mathrm{f}_{\mathcal{A}}\left(L^{*}, K\right) & =\mathrm{e}^{2 \pi i\left[L^{*}, K\right]_{\mathcal{A}}} & & (\text { by }(2.21)) \\
& =\mathrm{e}^{2 \pi i[L, K]_{\mathcal{B}}} & (\text { by Lemma 12.1) } \\
& =\mathrm{f}_{\mathcal{B}}(L, K) & & (\text { by }(2.21) .)
\end{array}
$$


(ii)

$$
\begin{aligned}
\ell_{\mathcal{A}}\left(L^{*}, d\right) & =\prod_{\substack{p \\
\left(\frac{d}{p}\right)=0}}\left(1+\frac{f_{a}\left(L^{*}, K_{p}\right)}{p}\right) \quad(\text { by }(2.33)) \\
& =\prod_{\substack{p \\
\left(\frac{d}{p}\right)=0}}\left(1+\frac{f_{\mathcal{B}}\left(L, K_{p}\right)}{p}\right) \quad(\text { by }(i)) \\
& =\ell_{\mathcal{B}}(L, d) \quad(\text { by }(2.33)) .
\end{aligned}
$$

(iii)

$$
\begin{aligned}
m_{a}\left(L^{*}, d\right) & =\lim _{s \rightarrow 1^{+}} \prod_{\substack{p \\
\left(\frac{d}{p}\right)=1}} \frac{\left(1-\frac{1}{p^{s}}\right)\left(1+\frac{1}{p^{s}}\right)}{\left(1-\frac{f_{\mathcal{A}}\left(L^{*}, K_{p}\right)}{p^{s}}\right)\left(1-\frac{f_{\mathcal{A}}\left(L^{*}, K_{p}\right)^{-1}}{p^{s}}\right)} \\
& =\lim _{s \rightarrow 1^{+}} \prod_{\substack{p \\
\left(\frac{d}{p}\right)=1}} \frac{\left(1-\frac{1}{p^{s}}\right)\left(1+\frac{1}{p^{s}}\right)}{\left(1-\frac{f_{\mathcal{B}}\left(L, K_{p}\right)}{p^{s}}\right)\left(1-\frac{f_{\mathcal{B}}\left(L, K_{p}\right)^{-1}}{p^{s}}\right)} \quad(\text { by }(i)) \\
& =m_{\mathcal{B}}(L, d) .
\end{aligned}
$$

Theorem 12.4 Thesum

$$
\sum_{\substack{L \in H(d) \\ L \neq 1}} f(L, K) \ell(L, d) m(L, d)
$$

does not depend upon the choice of basis $\mathcal{A}$.

Proof We have

$$
\begin{aligned}
& \sum_{\substack{L \in H(d) \\
L \neq 1}} f_{\mathcal{A}}(L, K) \ell_{\mathcal{A}}(L, d) m_{\mathcal{A}}(L, d) \\
& \quad=\sum_{\substack{L \in H(d) \\
L \neq 1}} f_{\mathcal{A}}\left(L^{*}, K\right) \ell_{\mathcal{A}}\left(L^{*}, d\right) m_{\mathcal{A}}\left(L^{*}, d\right) \quad \text { (by Lemma 12.2) } \\
& =\sum_{\substack{L \in H(d) \\
L \neq 1}} f_{\mathcal{B}}(L, K) \ell_{\mathcal{B}}(L, d) m_{\mathcal{B}}(L, d) \quad \text { (by Lemma 12.3). }
\end{aligned}
$$

Acknowledgement The second author would like to acknowledge the hospitality of $\mathrm{Mac}$ ac quarie University where the research for this paper was carried out in February and $\mathrm{M}$ arch of 1997. 


\section{References}

[1] H. Cohn, Advanced Number Theory. Dover Publications, Inc., New York, 1980.

[2] D. A. Cox, Primes of the Form $x^{2}+n y^{2}$. John Wiley and Sons, New York, 1989.

[3] I. S. Gradshteyn and I. M. Ryzhik, Table of Integrals, Series, and Products. Fifth Edition, Academic Press, 1994.

[4] G. H. Hardy and E. M. Wright, An Introduction to the Theory of Numbers. Oxford, 1960.

[5] J. G. Huard, P. Kaplan and K. S. Williams, The Chowla-Selberg formula for genera. Acta Arith. 73(1995), 271-301.

[6] P. Kaplan and K. S. Williams, On a formula of Dirichlet. Far East J. M ath. Sci. 5(1997), 153-157.

[7] W. Narkiewicz, Elementary and Analytic Theory of Algebraic N umbers. Springer-Verlag, New York, 1990.

[8] A. Selberg and S. Chowla, On Epstein's zeta-function. J. Reine Angew. M ath. 227(1967), 86-110.

[9] C. L. Siegel, Advanced Analytic Number Theory. Tata Institute of Fundamental Research, Bombay, 1980.

[10] K. S. Williams and N.-Y. Zhang, The Chowla-Selberg relation for genera. Preprint, 1993.

Centre for Number Theory Research

School of Mathematics, Physics,

Computing and Electronics

M acquarie University

Sydney, N SW

Australia 2109

email: alf@mpce.mq.edu.au
Centrefor Research in Algebra and

Number Theory

School of M athematics and Statistics

Carleton University

Ottawa, Ontario

K1S $5 B 6$

email: williams@math.carleton.ca 